\title{
NOTES ON MATRIX FACTORIZATIONS AND KNOT HOMOLOGY
}

\author{
A. OBLOMKOV
}

\begin{abstract}
These are the notes of the lectures delivered by the author at CIME in June 2018. The main purpose of the notes is to provide an overview of the techniques used in the construction of the triply graded link homology. The homology is space of global sections of a particular sheaf on the Hilbert scheme of points on the plane. Our construction relies on existence on the natural push-forward functor for the equivariant matrix factorizations, we explain the subtleties on the construction in these notes. We also outline a proof of the Markov moves for our homology as well as some explicit localization formulas for knot homology of a large class of links.
\end{abstract}

\section{INTRODUCTION}

The discovery of the knot homology [Kho00] of the links in the three-sphere, motivated search for the homological invariants of the three-manifolds. Heegard-Floer homology were discovered soon after Khovanov's seminal work, this homology categorifies the simplest case of WRT invariants (the invariants at the fourth root of unity). More general WRT invariants are beyond of the reach of currently available technique. Thus it is very important to reveal as much structure of the Khovanov homology as it is possible.

The mathematical construction of WRT invariants relies on special properties JW projectors at the root of unity, thus it is natural to search for the analogues of the projectors in the knot homology theory. If the algebraic variety is endowed with the action of the torus with the zero-dimensional locus, the algebraic geometry offer a natural decomposition of category of the coherent sheaves into the mutually orthogonal pieces [HL19], hence we have a natural analog of the JW projectors. In the paper [OR18d] we constructed a map from the braid group to the category of coherent sheaves on the free Hilbert scheme of points on the plane such that that Markov moves properties hold for the vector space of the global sections of the sheaf. Thus we have geometric candidate for JW projectors for such knot homology.

The quest for the geometric interpretation of JW projectors was the main motivation for the author of the notes to develop the connection between sheaves on the Hilbert scheme of points and knot homology. The localization type formulas were first encountered by the author in the joint work with Jake Rasmussen and Vivek Shende ORS18 where the homology of the torus knots were connected with the topology of the Hilbert schemes of points on the homogeneous plane singularities (see also GORS14]). However, back in 2012 it was a total mystery to the author how one would expand the relation in ORS18, GORS14 beyond the torus knots.

The connection was demystified by Lev Rozansky who was armed with the physics intuition as well as very deep understanding of already existing knot homology theories. As it

The work of A.O. was supported in part by the NSF CAREER grant DMS-1352398, the NSF FRG grant DMS-1760373 grant and Simons Foundation Fellowship No.561855. 
turned out the searched after knot homology has a natural interpretation within the framework of the Kapustin-Saulina-Rozansky topological quantum field theory for the cotangent bundles to the Lie algebras as targets [OR18a]. A purely mathematical theory underlying the physical predictions is laid out in the series of our joint papers OR18e, OR18c, [OR17, OR18b, OR18d]. To provide an introduction to the technique of these paper is the main goal of this note.

1.1. Main result. Let us state a consequence of the results from the papers that requires the minimal amount of new notations. We need some notations, though. Throughout the paper we use notation $D_{G}^{p e r}(X)$ for the derived category of the two-periodic $G$-equivariant complexes of coherent sheaves on $X$, where $G$ is a group acting on $X$. For us particularly important case of the pair $X, G$ is $\operatorname{Hilb}_{n}\left(\mathbb{C}^{2}\right), T_{s c}=\mathbb{C}^{*} \times \mathbb{C}^{*}$ with scaling action of $T_{s c}$ on $\mathbb{C}^{2}$. The dual $\mathcal{B}$ to the universal quotient bundle $\mathcal{B}^{\vee},\left.\mathcal{B}^{\vee}\right|_{I}=\mathbb{C}[x, y] / I$ will be used in our construction of the knot homology.

We also use notation $\mathfrak{B r}_{n}$ for the braid group on $n$ strands. For an element $\beta \in \mathfrak{B r}_{n}$ we can form a link in the three-sphere $L(\beta)$ by closing the braid in the most natural way.

Theorem 1.1.1. OR18d] There is a constructive procedure that assigns to a braid $\beta \in \mathfrak{B r}_{n}$ an object $S_{\beta} \in D_{T_{s c}}^{\text {per }}\left(\operatorname{Hilb}_{n}\left(\mathbb{C}^{2}\right)\right)$ such that

(1) $S_{\beta \cdot \mathrm{FT}}=S_{\beta} \otimes \operatorname{det}(\mathcal{B})$ where $\mathrm{FT}$ is the full twist on $n$ strands

(2) The triply graded vector space $\operatorname{HHH}(\beta):=H^{*}\left(S_{\beta} \otimes \Lambda^{\bullet} \mathcal{B}\right)$ is an isotopy invariant of the closure $L(\beta)$.

(3) The character of representation of the anti-diagonal torus $\mathbb{C}_{a}^{*} \subset T_{s c}$ on the spaces $H^{*}\left(S_{\beta} \otimes \Lambda^{i} \mathcal{B}\right)$ is the HOMFLYPT polynomial:

$$
\sum_{i} a^{i} \chi_{q}\left(\mathbb{C}_{a}^{*}, H^{*}\left(S_{\beta} \otimes \Lambda^{i} \mathcal{B}\right)\right)=\operatorname{HOMFLYPT}(L(\beta)) .
$$

The constructive procedure in the statement of theorem relies on the theory of matrix factorizations and in this note we try to present a gentle introduction into the aspects of the theory of matrix factorizations that are necessary for our theory. The author of the notes learned theory of matrix factorizations from discussions with Lev Rozansky, as result the exposition here is quite biased.

The first construction of the triply-graded categorification of the HOMFLYPT invariant appeared in the seminal work of Mikhail Khovanov and Lev Rozansky [KR08]. It is natural to conjecture that the homology discussed in these notes coincide with the Khovanov-Rozansky homology.

1.2. Outline. After defining and motivating the category of matrix factorizations in section 2.1.2 we spend some time discussing the most common type of matrix factorizations, Koszul matrix factorizations in section 2.2. The Koszul matrix factorizations are in many regards are analogous to the complete intersection rings and in this section we make this analogy more precise by providing a method for constructing a matrix factorization from a complete intersection (see lemma 2.2.3).

Next we discuss Knorrer periodicity in section 2.3 which is the most basic equivalence relation between the categories of matrix factorizations. After that we explain how one would perform push-forward and pull-back between the categories of matrix factorizations, see section 2.4. Finally, in the section 2.5 we introduce the equivariant matrix factorizations, in particular we explain the difference between the strongly and weakly equivariant matrix 
factorizations, later we only work with the weakly equivariant matrix factorizations since the weak equivariance allows us to define the equivariant push-forward.

In the section 3 we explain the key point of our construction, the homomorphism from the braid group $\mathfrak{B r}_{n}$ to the category of matrix factorizations. First in the section 3.1 we introduce our main space $\mathcal{X}$ with a potential $W$ and define a convolution algebra structure $\star$ on the category $\mathrm{MF}_{\mathrm{GL}_{n} \times B^{2}}(\mathcal{X}, W)$, here $B \subset \mathrm{GL}_{n}$ is a subgroup of upper-triangular matrices. There is a slightly smaller space $\overline{\mathcal{X}}$ with potential $\bar{W}$ such that Knorrer periodicity identifies $\mathrm{MF}_{\mathrm{GL}_{n} \times B^{2}}(\mathcal{X}, W)$ with $\mathrm{MF}_{B^{2}}(\overline{\mathcal{X}}, \bar{W})$ and it intertwines the convolution product $\star$ with the convolution product $\bar{\star}$, we provide details in the section 3.2 . After setting notations for the ordinary and affine braid groups in section 3.3 we state main properties of the homomorphisms:

$$
\Phi: \mathfrak{B r}_{n} \rightarrow \mathrm{MF}_{B^{2}}\left(\overline{\mathcal{X}}^{s t}, W\right), \quad \Phi^{a f f}: \mathfrak{B r}_{n}^{a f f} \rightarrow \operatorname{MF}_{B^{2}}(\overline{\mathcal{X}}, W),
$$

the pull-back along $j_{s t}: \mathcal{X}^{s t} \rightarrow \mathcal{X}$ intertwines these homomorphisms. We postpone the details of the construction of homomorphisms $\Phi, \Phi^{a f f}$ till section 5 .

In section 4 we explain how one can use the homomorphism $\Phi$ to construct the triplygraded homology. The free Hilbert scheme FHilb ${ }_{n}^{\text {free }}$ consists of the $B$-conjugacy classes $\mathrm{FHilb}_{n}^{\text {free }}=\widetilde{\mathrm{FHilb}}_{n}^{\text {free }} / B$ pairs of matrices with cyclic vector such that the monomials of the matrices applied to the vector span $\mathbb{C}^{n}$. There is an embedding of the $B$-cover $\widetilde{\mathrm{FHilb}}_{n}^{\text {free }}$ of the free Hilbert scheme into the stable version of our space $\widetilde{\mathrm{FHilb}}_{n}^{\text {free }} \rightarrow \overline{\mathcal{X}}^{s t}$ and we define the homology group:

$$
\mathbb{H}^{i}(\beta):=\mathbb{H}^{*}\left(\left(\Phi(\beta) \otimes \Lambda^{i} \mathcal{B}\right)^{B}\right),
$$

where $\mathcal{B}$ is the tautological vector bundle over the free Hilbert scheme. It is shown in OR18e that the graded dimension total sum

$$
\operatorname{HHH}(\beta)=\oplus_{i} H^{i}(\beta),
$$

is a triply graded knot invariant of the closure $L(\beta)$. We explain in the section 4.2 why this invariant specializes to the HOMFLYPT invariant after we forget about one of the gradings. Here $H^{i}(\beta)$ is $\mathbb{H}^{b+i}(\beta)$ with $b=b(\beta)$ being some specific function of $\beta$.

The free Hilbert scheme FHilb ${ }_{n}^{\text {free }}:=\widetilde{\text { FHilb }}_{n}^{\text {free }} / B$ is smooth and it contains the usual flag Hilbert scheme $\mathrm{FHilb}_{n} \subset \mathrm{FHilb}_{n}^{\text {free }}$ which is very singular and not even a local complete intersection. The relation of our homology with the honest flag Hilbert scheme is the following:

$$
\mathbb{S}_{\beta}=j_{e}(\Phi(\beta))^{B} \in D_{T_{s c}}^{\text {per }}\left(\text { FHilb }_{n}^{\text {free }}\right), \quad \operatorname{supp}\left(\mathcal{H}\left(\mathbb{S}_{\beta}\right)\right) \subset \text { FHilb }_{n},
$$

where $\mathcal{H}\left(\mathbb{S}_{\beta}\right)$ is the sheaf of FHilb ${ }_{n}^{\text {free }}$ which is the homology of the two-periodic complex $\mathbb{S}_{\beta}$.

The most non-trivial part of the statement from OR18e is the fact that the homology $\operatorname{HHH}(\beta)$ do not change under the Markov move that decreases the number of strands in the braid. In the section 7 we give a sketch of a proof the Markov move invariance, we rely in this section on the material of section 5 where the details of the construction of the braid group action are given.

In the section 6 we do a simplest computation in the convolution algebra of the category of matrix factorizations in the case $n=2$. We show that in $\operatorname{MF}_{B^{2}}\left(\overline{\mathcal{X}}^{s t}, \bar{W}\right)$ we have an isomorphism

$$
\mathcal{C}_{\bullet} \star \mathcal{C}_{\bullet} \simeq \mathbf{q}^{4} \mathcal{C}_{\bullet} \oplus \mathbf{q}^{2} \mathcal{C}_{\bullet}
$$


which is the geometric counter-part of the fact that the square of the non-trivial Soergel bimodule for $n=2$ is equal to a double of itself Soe01.

Finally, in the section 8 we define the categorical Chern functor:

$$
\mathrm{CH}_{l o c}^{s t}: \mathrm{MF}_{\mathrm{GL}_{n} \times B^{2}}\left(\mathcal{X}^{s t}, W\right) \rightarrow D_{T_{s c}^{p e r}}^{\text {per }}\left(\operatorname{Hilb}_{n}\left(\mathbb{C}^{2}\right)\right) .
$$

We also discuss the properties of the conjugate functor $\mathrm{HC}_{l o c}^{s t}$ (see OR18f] for the original construction )which is monoidal. The sheaf $S_{\beta}$ in the theorem 1.1.1 is given by:

$$
S_{\beta}=\mathrm{CH}_{l o c}^{s t}(\Phi(\beta)) \text {. }
$$

The advantage of the sheaf $S_{\beta}$ over $\mathbb{S}_{\beta}$ is that it is a $T_{s c}$-equivariant periodic complex of sheaves on the smooth manifold $\operatorname{Hilb}_{n}\left(\mathbb{C}^{2}\right)$ thus we can hope to use $T_{s c}$-localization technique for computation of the knot homology. There are some technical issues with using the localization method directly as we discuss in 8.5. We also explain how these technical issue could be circumvented and in particular how one can apply this technique to compute the homology of the sufficiently positive elements of Jucy-Murphy algebra. This formula was conjectured in GRN16.

1.3. Other results. We also would like to mention that many relevant aspects of matrix factorizations are not covered in these notes. The reader could consult papers the original papers of Orlov for the connections with mirror symmetry Orl04] and paper Dyc11 for some further discussion of the foundations of the theory of matrix factorizations and of course the seminal paper of Khovanov and Rozansky KR08 where the first construction of a triply graded homology of the links was proposed. The constructions in these notes are motivated by the physical theory of Kapustin, Saulina, Rozansky [KRS09], the reader is encouraged to read wonderful, basically purely mathematical paper [KR10] where the role of matrix factorizations in the theory is explained.

Let us also mention that there is a slightly different perspective on the geometric interpretation of the knot homology due to Gorsky, Negut, Hogencamp and Rasmussen [GRN16], [GH17]. Their approach takes the theory of Soergel bimodules and the corresponding link homology construction [KR08] as a starting point of theory, rather than the categories of matrix factorizations discussed in these notes. Finally, let us mention the recent work of Hogencamp and Elias on categorical diagonalization [EH16, [EH17a, [EH17b] which provides a categorical setting for the localozation in the category of coherent sheaves.

These notes by no means were intended as a comprehensive survey of the theory of matrix factorization or of the theory of knot homology. It is a merely is a slightly extended version of the three lectures that the author delivered at 2018 CIME. Thus the author asks for an apology from the colleagues whose contributions to the fields are not covered in the notes.

Acknowledgments: First of all I would like to thank my coathor and friend Lev Rozansky for teaching everything that is in these notes. All results in these notes are contained in our joint papers. I also would like to thank Andrei Negut and Tina Kanstrup for discussion related to the content of the notes. I am very grateful to an anonymous referee for many great suggestion on improving the first draft of the notes. I am very greatful to CIME foundation for opportunity to teach the course at the Summer school. The participants of the course provided valuable feed-back on the material. I was also partially supported by NSF and Simons foundation. 


\section{MATRIX FACTORIZATIONS}

In this section we remind some basic facts about matrix factorizations. There are many excellent exposition on matrix factorizations [Eis80, Orl04, Dyc11 and we choose not to concentrate on usual matrix factorizations, instead we aim to define equivariant matrix factorizations and subtleties that arise in attempt to define such. We also discuss Koszul matrix factorizations and the (equivariant) push-forward functor from OR18e.

2.1. Motivation and examples. Given an affine variety $\mathcal{Z}$ and a function $F$ on it we define Eis80 the homotopy category $\operatorname{MF}(\mathcal{Z}, F)$ of matrix factorizations whose objects are complexes of projective $R=\mathbb{C}[\mathcal{Z}]$-modules $M^{0}, M^{1} M=M^{0} \oplus M^{1}$ equipped with the differential

$$
D=\left(D^{0}, D^{1}\right) \in \operatorname{Hom}_{R}\left(M^{0}, M^{1}\right) \oplus \operatorname{Hom}_{R}\left(M^{1}, M^{0}\right)
$$

such that $D^{2}=F$. Thus $\operatorname{MF}(\mathcal{Z}, F)$ is a triangulated category as explained in subsection 3.1 of [Orl04]. We first discuss the objects of this category, then discuss various properties of the morphism spaces.

It is convenient to think about a matrix factorization $\left(M^{0} \oplus M^{1}, D\right)$ as two-periodic curved complex:

$$
\ldots \stackrel{D^{1}}{\longrightarrow} M^{0} \stackrel{D^{0}}{\longrightarrow} M^{1} \stackrel{D^{1}}{\longrightarrow} M^{0} \stackrel{D^{0}}{\longrightarrow} M^{1} \stackrel{D^{1}}{\longrightarrow} \ldots, \quad D^{2}=F .
$$

Let us look at several basic examples of matrix factorizations and discuss briefly a motivation for the definition of the matrix factorizations by Eisenbud [Eis80].

Example 2.1.1. $\mathcal{Z}=\mathbb{C}, R=\mathbb{C}[x]$ and $F=x^{5}$. The two-periodic complex

$$
\ldots \stackrel{x^{2}}{\longrightarrow} \underline{R} \stackrel{x^{3}}{\longrightarrow} R \stackrel{x^{2}}{\rightarrow} R \stackrel{x^{3}}{\rightarrow} R \stackrel{x^{2}}{\longrightarrow} \ldots
$$

is an example of an object in $\operatorname{MF}\left(\mathbb{C}, x^{5}\right)$. Here and everywhere below we underline to indicate zeroth homological degree.

Example 2.1.2. $\mathcal{Z}=\mathbb{C}^{2}, R=\mathbb{C}[x, y], F=x y$. The two-periodic complex

$$
\ldots \stackrel{x}{\rightarrow} \underline{R} \stackrel{y}{\rightarrow} R \stackrel{x}{\rightarrow} R \stackrel{y}{\rightarrow} R \stackrel{x}{\rightarrow} \ldots
$$

is an example of an object in $\operatorname{MF}\left(\mathbb{C}^{2}, x y\right)$.

The last example has the following geometric interpretation. A module over a quotient ring $Q=\mathbb{C}[x, y] /(x y)$, in general, does not have a finite free resolution. In particular, $M=$ $\mathbb{C}[x]=Q /(y)$ is a module over $Q$ with an infinite free resolution:

$$
0 \leftarrow M \stackrel{y}{\longleftarrow} Q \stackrel{x}{\leftarrow} Q \stackrel{y}{\leftarrow} Q \stackrel{x}{\leftarrow} \ldots
$$

This resolution has a two-periodic (half-infinite) tail which is a reduction of the matrix factorization from the example 2.1.2. As explained in Eis80 this phenomenon is more general.

We felt obliged to mention these results on matrix factorizations to honor the origins of the subjects. For further development of Eisenbud theory the reader is encouraged to look at [Eis80, as well as Orl04, Orl09, Orl11 where the connection with B-model theory is developed. However, the hypersurfaces defined by the potentials from OR18e do not have a clear geometric interpretation and it is unclear to us how to make use of Eisenbud's theory in our case. Instead, more elementary homological aspect of the matrix factorizations is important to us. Roughly stated, the very important observation is that all important homological information about the category of matrix factorizations is contained in a neighborhood of the critical locus. We explain more rigorous statement below. 
It is a good place to define morphisms in the category of matrix factorizations. Suppose we have two objects $\mathcal{F}_{1}=\left(M_{1}, D_{1}\right), \mathcal{F}_{2}=\left(M_{2}, D_{2}\right) \in \operatorname{MF}(\mathcal{Z}, F)$ then we define:

$$
\underline{\operatorname{Hom}}\left(\mathcal{F}_{1}, \mathcal{F}_{2}\right):=\left\{\Psi \in \operatorname{Hom}_{R}\left(M_{1}, M_{2}\right) \mid \Psi \circ D_{1}=D_{2} \circ \Psi\right\} .
$$

Since the modules $M_{i}$ are $\mathbb{Z}_{2}$-graded we have a decomposition

$$
\underline{\operatorname{Hom}}\left(M_{1}, M_{2}\right)=\oplus_{i \in \mathbb{Z}_{2}} \underline{\operatorname{Hom}}^{i}\left(M_{1}, M_{2}\right)
$$

where $\underline{\operatorname{Hom}}^{i}\left(M_{1}, M_{2}\right) \subset \operatorname{Hom}_{R}^{i}\left(M_{1}, M_{2}\right):=\operatorname{Hom}_{R}\left(M_{1}^{0}, M_{2}^{i}\right) \oplus \operatorname{Hom}_{R}\left(M_{1}^{1}, M_{2}^{i+1}\right)$.

We say that an element $\Psi \in \underline{\operatorname{Hom}}^{0}\left(\mathcal{F}_{1}, \mathcal{F}_{2}\right)$ is homotopic to zero: $\Psi \sim 0$ if there is $h \in$ $\operatorname{Hom}^{1}\left(M_{1}, M_{2}\right)$ such that $\Psi=h \circ D_{1}+D_{2} \circ h$. Finally, we define the space of morphisms as a set of equvalence classes with respect to the homotopy equivalence:

$$
\operatorname{Hom}\left(\mathcal{F}_{1}, \mathcal{F}_{2}\right):=\underline{\operatorname{Hom}}^{0}\left(\mathcal{F}_{1}, \mathcal{F}_{2}\right) / \sim
$$

Now that we defined the objects and morphisms between the objects we can state Orlov's theorem

Theorem 2.1.3. Orl04 $\operatorname{MF}(\mathcal{Z}, F)$ has a structure of the triangulated category.

To complete our discussion of the homological properties of category of matrix factorizations with respect to their critical locus let us observe that an element $f \in R$ naturally gives an element of $\operatorname{Hom}(\mathcal{F}, \mathcal{F})$. For simplicity let us also assume that $\mathcal{Z} \subset \mathbb{C}^{m}$. Then we have a well-defined ideal $I_{c r i t} \subset R$ generated by $\frac{\partial F}{\partial x_{i}} i=1, \ldots, m$ and $x_{i}$ are coordinates on $\mathbb{C}^{m}$.

Proposition 2.1.4. For any $\mathcal{F} \in \operatorname{MF}(\mathcal{Z}, F)$ and $f \in I_{\text {crit }}$ we have:

$$
\underline{\operatorname{Hom}}^{0}(\mathcal{F}, \mathcal{F}) \ni f \sim 0 .
$$

Proof. If it is enough to show the statement for $f=\frac{\partial F}{\partial x_{i}}$. Thus the statement follows since:

$$
\frac{\partial F}{\partial x_{i}}=\frac{\partial D}{\partial x_{i}} D+D \frac{\partial D}{\partial x_{i}}
$$

and $\frac{\partial D}{\partial x_{i}}$ provides the needed homotopy.

The last proposition implies that category of matrix factorizations is model for the coherent sheaves on possibly singular critical locus of the potential $F$. When the potential is linear in by some set of variables than there is an equivalence between with the DG category of the critical locus (see section 8.3 for more discussion). Another manifestation of this principle is the shrinking lemma, see lemma 3.4 .2 below.

2.2. Koszul matrix factorizations. The matrix factorizations from examples 2.1 .2 and 2.1.1 are examples of so called Koszul matrix factorizations which we discuss in this subsection. Suppose we have a presentation of the potential as $\operatorname{sum} F=\sum_{i=1}^{n} a_{i} b_{i}$. Then we define Koszul matrix factorization $\mathrm{K}[\vec{a}, \vec{b}] \in \mathrm{MF}(\mathcal{Z}, F)$ as

$$
\mathrm{K}[\vec{a}, \vec{b}]:=\left(\Lambda^{\bullet} V, D\right), \quad D=\sum_{i} a_{i} \theta_{i}+b_{i} \frac{\partial}{\partial \theta_{i}},
$$

where $V=\left\langle\theta_{1}, \ldots, \theta_{n}\right\rangle$. The examples 2.1.1, 2.1.2 are $\mathrm{K}\left[x^{2}, x^{3}\right]$ and $\mathrm{K}[x, y]$, respectively.

The Koszul matrix factorizations are tensor products of the simplest Koszul matrix factorizations. Indeed, given two matrix factorizations $\mathcal{F}_{1} \in \operatorname{MF}\left(\mathcal{Z}, F_{1}\right), \mathcal{F}_{2} \in \operatorname{MF}\left(\mathcal{Z}, F_{2}\right)$ the tensor product $\mathcal{F}_{1} \otimes \mathcal{F}_{2} \in \operatorname{MF}\left(\mathcal{Z}, F_{1}+F_{2}\right)$ is the matrix factorization $\left(M_{1} \otimes M_{2}, D_{1} \otimes 1+1 \otimes D_{2}\right)$. Thus we have $\mathrm{K}[\vec{a}, \vec{b}]=\bigotimes_{i=1}^{n} \mathrm{~K}\left[a_{i}, b_{i}\right]$. 
An object of the category of matrix factorizations with the zero potential is a two-periodic complex of coherent sheaves. We denote by $D^{\text {per }}(\mathcal{Z})$ the derived category of the twoperiodic complexes of coherent sheaves. Given two matrix factorizations $\mathcal{F}_{1} \in \operatorname{MF}(\mathcal{Z}, F)$, $\mathcal{F}_{2} \in \operatorname{MF}(\mathcal{Z},-F)$ their tensor product is an element of $D^{\text {per }}(\mathcal{Z})$ and proposition 2.1.4 implies:

Corollary 2.2.1. For $\mathcal{F}_{1} \in \operatorname{MF}(\mathcal{Z}, F), \mathcal{F}_{2} \in \operatorname{MF}(\mathcal{Z},-F)$ homology of the two-periodic complex $\mathcal{F}_{1} \otimes \mathcal{F}_{2}$ are supported on the zero locus of $I_{\text {crit }}$.

Now let us discuss a method for constructing interesting Koszul matrix factorizations. Let us first recall some basic properties of the usual Koszul complexes. The sequence $f_{1}, \ldots, f_{m} \in$ $R$ is called regular if $f_{i}$ is not a zero-divisor in the quotient $R /\left(f_{1}, \ldots, f_{i-1}\right)$ for $i=1, \ldots, n$. It is known that the regularity does not depend on the order of the elements. There is an equivalent way to define regularity with the help of Koszul complexes. The Koszul complex of $\vec{f}$ is:

$$
\mathrm{K}[\vec{f}]=\left(\Lambda^{\bullet} V, D\right), \quad D=\sum_{i} f_{i} \frac{\partial}{\partial \theta_{i}}
$$

Proposition 2.2.2. The sequence $\left(f_{1}, \ldots, f_{m}\right)$ is regular if and only if:

$$
H^{i}(\mathrm{~K}[\vec{f}])=0, \quad i>0, \quad, \quad H^{0}(\mathrm{~K}(\vec{f}))=R /\left(f_{1}, \ldots, f_{m}\right) .
$$

Given a finite complex of $\left(C_{\bullet}, d\right)$ of free $R$-modules we denote by $\left[C_{\bullet}\right]_{\text {per }}$ the two-periodic folding of the complex. It is an element of $\operatorname{MF}(\mathcal{Z}, 0)$. Suppose $F \in\left(f_{1}, \ldots, f_{m}\right)$ and the sequence $\vec{f}$ is regular. Then the lemma below shows that there is an essentially unique way to deform the complex $[\mathrm{K}[\vec{f}]]_{\text {per }}$ to an element of $\operatorname{MF}(\mathcal{Z}, F)$. We outline a proof of the lemma to demonstrate the key deformation theory technique that is used in many constructions of OR18e].

Lemma 2.2.3. Suppose $F \in\left(f_{1}, \ldots, f_{m}\right)$ and the sequence $\vec{f}$ is regular. Then the Koszul complex

$$
C_{\bullet}=\mathrm{K}[\vec{f}]=\left\{C_{0} \stackrel{d_{1}^{+}}{\longleftarrow} C_{1} \stackrel{d_{2}^{+}}{\longleftarrow} \ldots \stackrel{d_{m}^{+}}{\longleftarrow} C_{m}\right\}
$$

could be completed with the opposite differentials $d_{i}^{-}: C_{\bullet} \rightarrow C_{\bullet}+2 i-1, i>0$ such that

$$
\left(C_{\bullet}, d^{+}+d^{-}\right) \in \operatorname{MF}(\mathcal{Z}, F) \text {. }
$$

Proof. We will construct the differentials $d_{i}$ iteratively. Since the sequence is regular we have a homotopy equivalence:

$$
\left(C_{\bullet}, d^{+}\right) \sim Q=R /\left(f_{1}, \ldots, f_{m}\right) .
$$

Let us also introduce notation for the graded pieces of the space of homomorphisms:

$$
\operatorname{Hom}^{i}\left(C_{\bullet}, C_{\bullet}\right)=\oplus_{j} \operatorname{Hom}\left(C_{j}, C_{-i+j}\right) .
$$

The element $F$ is an endomorphism of $\left(C_{\bullet}, d^{+}\right)$and because of (2.1) it is homotopic to zero by the lemma assumptions. Thus there is a homotopy $h^{(-1)} \in \operatorname{Hom}^{-1}\left(C_{\bullet}, C_{\bullet}\right)$ such that $F=h^{(1)} \circ d^{+}+d^{+} \circ h^{(1)}$. Let us set $D^{(1)}=d^{+}+h^{(1)}$.

The differential $D^{(1)}$ is the first order approximation for our desired extension. It is not differential of a matrix factorization if $n>1$ since:

$$
\left(D^{(1)}\right)^{2}=F+\left(h^{(1)}\right)^{2} .
$$


However the correction term $\left(h^{(1)}\right)^{2}$ is actually an element of $\operatorname{Hom}_{d^{+}}^{-2}\left(C_{\bullet}, C_{\bullet}\right)$, that is it commutes with the differential $d^{+}$:

$d^{+} \circ h^{(1)} \circ h^{(1)}=F h^{(1)}-h^{(1)} \circ d^{+} \circ h^{(1)}=F h^{(1)}+h^{(1)} \circ h^{(1)} \circ d^{+}-h^{(1)} F=h^{(1)} \circ h^{(1)} \circ d^{+}$.

Thus again by (2.1) there is a homotopy $h^{(3)} \in \operatorname{Hom}^{-3}\left(C_{\bullet}, C_{\bullet}\right)$ such that $h^{(1)}=d_{+} \circ h^{(3)}+$ $h^{(3)} \circ d_{+}$. We define the next approximation to the needed differential $D^{(3)}=d^{+}+h^{(1)}+h^{(3)}$. Again $D^{(3)}$ is not a differential of a matrix factorization if $n>3$ :

$$
\left(D^{(1)}+h^{(3)}\right)^{2}=F+\left(h^{(1)}\right)^{2}+D^{(1)} \circ h^{(3)}+h^{(3)} \circ D^{(1)}+\left(h^{(3)}\right)^{2}=F+h^{(1)} \circ h^{(3)}+h^{(1)} \circ h^{(3)}+\left(h^{(3)}\right)^{2} .
$$

The correction term belongs to $\operatorname{Hom}^{<-3}\left(C_{\bullet}, C_{\bullet}\right)$ and the degree four piece of this term is $h^{(1)} \circ h^{(3)}+h^{(3)} \circ h^{(1)}$. Let us check that $h^{(1)} \circ h^{(3)}+h^{(3)} \circ h^{(1)} \in \operatorname{Hom}_{d^{+}}^{-4}\left(C_{\bullet}, C_{\bullet}\right)$ :

$$
\begin{gathered}
d^{+} \circ h^{(1)} \circ h^{(3)}=F h^{(3)}-h^{(1)} \circ d^{+} \circ h^{(3)}=F h^{(3)}-\left(h^{(1)}\right)^{3}-h^{(1)} \circ h^{(3)} \circ d^{+}, \\
d^{+} \circ h^{(3)} \circ h^{(1)}=h^{(1)} \circ h^{(1)} \circ h^{(1)}-h^{(3)} \circ d^{+} \circ h^{(1)}=\left(h^{(1)}\right)^{3}-h^{(3)} F-h^{(3)} \circ h^{(1)} \circ d^{+} .
\end{gathered}
$$

By the same argument as before homomorphism $h^{(1)} \circ h^{(3)}+h^{(3)} \circ h^{(1)}$ is homotopic to zero and let denote by $h^{(5)} \in \operatorname{Hom}^{-5}\left(C_{\bullet}, C_{\bullet}\right)$. The next approximation for our differential is $D^{(5)}=d^{+}+h^{(1)}+h^{(3)}+h^{(5)}$ and

$$
\left(D^{(5)}\right)^{2}-F \in \operatorname{Hom}^{<-5}\left(C_{\bullet}, C_{\bullet}\right) .
$$

Similar method could be applied to show that correction term of degree six is homotopic to zero and thus we have the next order correction. Clearly, this iterative procedure terminates since our complex is of finite length. More formal proof of the lemma is given in lemma 2.1 in OR18e.

Remark 2.2.4. The only assumption on the complex $\left(C_{\bullet}, d^{+}\right)$that we used is that

$$
\operatorname{Hom}_{d^{+}}^{<0}\left(C_{\bullet}, C_{\bullet}\right) \sim 0 .
$$

Thus we can strengthen our lemma a little bit by replacing regularity Koszul complex by the condition (2.2)

It is natural to ask how canonical is the matrix factorization $\left(C_{\bullet}, d^{+}+d^{-}\right)$constructed in the previous lemma. Clearly, our method relies on a existence of various homotopies which are not unique. However, one can show that outcome of the iterative procedure in the proof is unique up to an isomorphism. We invite reader to try to apply the iterative method of the previous lemma to show lemma below, a formal proof could be found in lemma 3.7 in OR18e.

Lemma 2.2.5. Let $\left(C_{\bullet}, d^{+}\right)$be a complex of free modules with non-trivial terms in degrees from 0 to $l \geqslant 0$ such that $\operatorname{Hom}_{d^{+}}^{<0}\left(C_{\bullet}, C_{\bullet}\right) \sim 0$. Suppose we have two matrix factorizations

$$
\mathcal{F}=\left(C_{\bullet}, d^{+}+d^{-}\right), \tilde{\mathcal{F}}=\left(C_{\bullet}, d^{+}+\tilde{d}^{-}\right) \in \operatorname{MF}(\mathcal{Z}, F),
$$

where $d^{-}=\sum_{i \geqslant 0} d_{i}^{-}, \tilde{d}^{-}=\sum_{i \geqslant 0} \tilde{d}_{i}^{-}, d_{i}^{-}, \tilde{d}_{i}^{-} \in \operatorname{Hom}^{-2 i-1}\left(C_{\bullet}, C_{\bullet}\right)$ and $F \sim 0$ as endomorphism of $\left(C_{\bullet}, d^{+}\right)$. Then there is $\Psi=1+\sum_{i>0} \Psi_{i}, \Psi_{i} \in \mathrm{Hom}^{-i}\left(C_{\bullet}, C_{\bullet}\right)$ such that

$$
\Psi \circ\left(d^{+}+d^{-}\right) \circ \Psi^{-1}=d^{+}+\tilde{d}^{-} .
$$

Because of the previous lemma we will use notation $\mathrm{K}^{F}\left(f_{1}, \ldots, f_{m}\right) \in \operatorname{MF}(\mathcal{Z}, F)$ for a matrix factorization from the lemma 2.2.3. 
2.3. Knorrer periodicity. The critical locus of the potential $F=x y$ is a point $x=y=0$ so according to our principle we expect that the category of matrix factorizations with the potential $x y$ is equivalent to the category of matrix factorizations on the point. It is indeed the case and the equivalence is known under the name Knorrer periodicity and we explain the details below.

Let us denote the Koszul matrix factorization $\mathrm{K}[x, y] \in \mathrm{MF}\left(\mathbb{C}^{2}, x y\right)$ by $\mathrm{K}$. Then there is an exact functor between triangulated categories:

$$
\Phi: \operatorname{MF}(\mathrm{pt}, 0) \rightarrow \operatorname{MF}\left(\mathbb{C}^{2}, x y\right), \quad(M, D) \mapsto(M \otimes \mathbb{C}[x, y], D) \otimes \mathrm{K} .
$$

The functor in the inverse direction is the restriction functor:

$$
\Psi: \operatorname{MF}\left(\mathbb{C}^{2}, x y\right) \rightarrow \operatorname{MF}(\mathrm{pt}, 0), \quad(M, D) \mapsto\left(\left.M\right|_{x=0},\left.D\right|_{x=0}\right) .
$$

These functors are mutually inverse. Indeed, to show that $\Psi \circ \Phi=1$ we observe that $\left.\mathrm{K}\right|_{x=0}=[\mathbb{C}[y] \stackrel{y}{\leftarrow} \mathbb{C}[y]]$ which is a sky-scarper at $y=0$. We leave it as an exercise to a reader to show $\Phi \circ \Psi=1$.

More generally, if $\mathcal{Z}=\mathcal{Z}_{0} \times \mathbb{C}_{x, y}^{2}$ and $F_{0} \in \mathbb{C}\left[\mathcal{Z}_{0}\right]$ then there is a functor: $\Phi: \operatorname{MF}\left(\mathcal{Z}_{0}, F_{0}\right) \rightarrow$ $\operatorname{MF}\left(\mathcal{Z}, F_{0}+x y\right)$ given by tensoring with the Koszul complex $\mathrm{K}[x, y]$.

Theorem 2.3.1. Orl04] The functor $\Phi$ is an equivalence of triangulated categories.

2.4. Functoriality. Now we will use previously developed technique to define the pushforward functor for matrix factorizations. First we discuss a construction of the push-forward for embedding map: $j: \mathcal{Z}_{0} \hookrightarrow \mathcal{Z}$ where $\mathcal{Z}=\operatorname{Spec}(S)$ and $\mathcal{Z}_{0}=\operatorname{Spec}(R), R=S / I$.

Theorem 2.4.1. OR18e Suppose we have $F \in S, F_{0}=j^{*}(F)$ and $I=\left(f_{1}, \ldots, f_{m}\right)$ where $f_{i}$ form a regular sequence. Then there is well-defined functor of triangulated categories:

$$
j_{*}: \operatorname{MF}\left(\mathcal{Z}_{0}, F_{0}\right) \rightarrow \operatorname{MF}(\mathcal{Z}, F)
$$

Given an element $\mathcal{F}=(M, D) \in \operatorname{MF}\left(\mathcal{Z}_{0}, F_{0}\right)$ let us explain the construction of the element $j_{*}(\mathcal{F})=\widetilde{F} \in \operatorname{MF}(\mathcal{Z}, F)$. Since $M=R^{n}$ for some $n$ we can lift it to the module $\widetilde{M}=S^{n}$ as well as the differential to a $\mathbb{Z}_{2}$-graded endomorphism $\widetilde{D} \in \operatorname{Hom}_{S}\left(S^{n}, S^{n}\right),\left.\widetilde{D}\right|_{\mathcal{Z}_{0}}=D$. Since $f_{i}$ form a regular sequence we can form Koszul complex $\mathrm{K}\left(f_{1}, \ldots, f_{m}\right)=\left(\Lambda^{\bullet} \mathbb{C}^{n} \otimes S, d_{K}\right)$ which is a resolution of $S$-module $R$. The technique similar to the method of lemma 2.2.3 yields

Lemma 2.4.2. OR18e] There are $d_{i j}: \widetilde{M} \otimes \Lambda^{\bullet} \mathbb{C}^{n} \otimes S, i-j \in 1+\mathbb{Z}_{\geqslant 0}$ such that

$$
\widetilde{\mathcal{F}}=\left(\Lambda^{\bullet} \mathbb{C}^{n} \otimes S, d_{K}+\widetilde{D}+d^{-}\right) \in \operatorname{MF}(\mathcal{Z}, F)
$$

and the element $\widetilde{\mathcal{F}}$ is unique up to isomorphism.

To complete proof of the theorem 2.4.1 we need to show that the construction of $j^{*}$ extends to the spaces of the morphisms between the objects and to the space of homotopies between the morpshism, it is shown in lemma 3.7 of OR18e and we refer interested reader there for the technical details.

Unlike push-forward the pull-back functor is rather elementary. Suppose we have $f: \mathcal{Z} \rightarrow$ $\mathcal{Z}_{0}$ a morphism of affine varieties and $F=f^{*}\left(F_{0}\right), F_{0} \in \mathbb{C}\left[\mathcal{Z}_{0}\right]$. Since pull-back of a free module is free, we have a well-defined functor:

$$
f^{*}: \operatorname{MF}\left(\mathcal{Z}_{0}, F_{0}\right) \rightarrow \operatorname{MF}(\mathcal{Z}, F) .
$$

Finally, let us remark that the above defined pull-back and push-forward functors satisfy the base change relation for commuting squares of maps. 
2.5. Equivariant matrix factorizations. Matrix factorization is a natural object attached to a function on the affine manifold. However limiting yourself to only affine manifold is frustrating, so one would like to develop a theory of matrix factorizations on quasi-projective manifolds. There are some proposals in the literature for such theory, see for example [PV11].

In our work OR18e we chose an approach that is probably more limited than the one from PV11 but has an advantage of being computation friendly. So in OR18e to explore matrix factorizations on the manifolds that are group quotients of the affine manifolds, we develop theory of equivariant matrix factorizations. In this section we motivate our definitions and outline the ingredients of the construction from [OR18e].

Suppose the affine manifold $\mathcal{Z}$ has an action of an algebraic group $H$ and $F \in \mathbb{C}[\mathcal{Z}]^{H}$. Then one can say the matrix factorization $\mathcal{F}=(M, D) \in \operatorname{MF}(\mathcal{Z}, F)$ is strongly $G$-equivariant if $M$ is endowed with $H$-representation structure and the differential $D$ is $H$-equivariant. Let us denote the set of strongly equivariant matrix factorizations by $\operatorname{MF}_{H}^{s t r}(\mathcal{Z}, F)$. By requiring the morphism between the objects and the homotopies between the morphisms to be $H$ equivariant we can provide $\operatorname{MF}_{H}^{s t r}(\mathcal{Z}, F)$ with the structure of the triangulated category.

However, the notion of strong equivariance turns out to be too restrictive. Indeed, one of the key tools in our arsenal is the extension lemma 2.2 .3 together with the push-forward functor. So we would like to have analog of lemma 2.2.3 in the equivariant setting, for the $H$-equivariant ideal $I=\left(f_{1}, \ldots, f_{m}\right)$ with $f_{i}$ forming a regular sequence. Unfortunately, the proof of the lemma fails in the strongly equivariant setting because we can not guarantee that the homotopies in the iterative construction of proof are equivariant. If $H$ is reductive, we can save the proof by averaging along the maximal compact subgroup of $H$. But for non-reductive group we need a weaker notion of equivariance that relies on the Chevalley-Eilenberg complex explained below.

Let $\mathfrak{h}$ be the Lie algebra of $H$. Chevalley-Eilenberg complex $\mathrm{CE}_{\mathfrak{h}}$ is the complex $\left(V_{\bullet}(\mathfrak{h}), d\right)$ with $V_{p}(\mathfrak{h})=U(\mathfrak{h}) \otimes_{\mathbb{C}} \Lambda^{p} \mathfrak{h}$ and differential $d_{c e}=d_{1}+d_{2}$ where:

$$
\begin{gathered}
d_{1}\left(u \otimes x_{1} \wedge \cdots \wedge x_{p}\right)=\sum_{i=1}^{p}(-1)^{i+1} u x_{i} \otimes x_{1} \wedge \cdots \wedge \hat{x}_{i} \wedge \cdots \wedge x_{p}, \\
d_{2}\left(u \otimes x_{1} \wedge \cdots \wedge x_{p}\right)=\sum_{i<j}(-1)^{i+j} u \otimes\left[x_{i}, x_{j}\right] \wedge x_{1} \wedge \cdots \wedge \hat{x}_{i} \wedge \cdots \wedge \hat{x}_{j} \wedge \cdots \wedge x_{p},
\end{gathered}
$$

Let us denote by $\Delta$ the standard map $\mathfrak{h} \rightarrow \mathfrak{h} \otimes \mathfrak{h}$ defined by $x \mapsto x \otimes 1+1 \otimes x$. Suppose $V$ and $W$ are modules over the Lie algebra $\mathfrak{h}$ then we use notation $V \stackrel{\Delta}{\otimes} W$ for the $\mathfrak{h}$-module which is isomorphic to $V \otimes W$ as a vector space, the $\mathfrak{h}$-module structure being defined by $\Delta$. Respectively, for a given $\mathfrak{h}$-equivariant matrix factorization $\mathcal{F}=(M, D)$ we denote by $\mathrm{CE}_{\mathfrak{h}} \otimes \overrightarrow{\mathcal{F}}$ the h-equivariant matrix factorization $\left(C E_{\mathfrak{h}} \stackrel{\Delta}{\otimes} \mathcal{F}, D+d_{c e}\right)$. The h-equivariant structure on $\mathrm{CE}_{\mathfrak{h}} \stackrel{\Delta}{\otimes} \mathcal{F}$ originates from the left action of $U(\mathfrak{h})$ that commutes with right action on $U(\mathfrak{h})$ used in the construction of $\mathrm{CE}_{\mathfrak{h}}$.

A slight modification of the standard fact that $\mathrm{CE}_{\mathfrak{h}}$ is the resolution of the trivial module implies that $\mathrm{CE}_{\mathfrak{h}} \stackrel{\Delta}{\otimes} M$ is a free resolution of the $\mathfrak{h}$-module $M$.

Now we about to define a new category whose objects we refer to as weakly equivariant matrix factorizations. The objects of this category $\operatorname{MF}_{\mathfrak{h}}(\mathcal{Z}, W)$ are triples:

$$
\mathcal{F}=(M, D, \partial), \quad(M, D) \in \operatorname{MF}(\mathcal{Z}, W)
$$


where $M=M^{0} \oplus M^{1}$ and $M^{i}=\mathbb{C}[\mathcal{Z}] \otimes V^{i}, V^{i} \in \operatorname{Mod}_{\mathfrak{h}}, \partial \in \oplus_{i>j} \operatorname{Hom}_{\mathbb{C}[\mathcal{Z}]}\left(\Lambda^{i} \mathfrak{h} \otimes M, \Lambda^{j} \mathfrak{h} \otimes M\right)$ and $D$ is an odd endomorphism $D \in \operatorname{Hom}_{\mathbb{C}[\mathcal{Z}]}(M, M)$ such that

$$
D^{2}=F, \quad D_{t o t}^{2}=F, \quad D_{t o t}=D+d_{c e}+\partial,
$$

where the total differential $D_{\text {tot }}$ is an endomorphism of $\mathrm{CE}_{\mathfrak{h}} \stackrel{\Delta}{\otimes} M$, that commutes with the $U(\mathfrak{h})$-action.

Note that we do not impose the equivariance condition on the differential $D$ in our definition of matrix factorizations. On the other hand, if $\mathcal{F}=(M, D) \in \operatorname{MF}^{s t r}(\mathcal{Z}, F)$ is a matrix factorization with $D$ that commutes with $\mathfrak{h}$-action on $M$ then $(M, D, 0) \in \mathrm{MF}_{\mathfrak{h}}(\mathcal{Z}, F)$.

There is a forgetful map for the objects of the categories $\operatorname{Ob}\left(\operatorname{MF}_{\mathfrak{h}}(\mathcal{Z}, F)\right) \rightarrow \operatorname{Ob}(\operatorname{MF}(\mathcal{Z}, F)$ that forgets about the correction differentials:

$$
\mathcal{F}=(M, D, \partial) \mapsto \mathcal{F}^{\sharp}:=(M, D) .
$$

Given two $\mathfrak{h}$-equivariant matrix factorizations $\mathcal{F}=(M, D, \partial)$ and $\tilde{\mathcal{F}}=(\tilde{M}, \tilde{D}, \tilde{\partial})$ the space of morphisms $\operatorname{Hom}(\mathcal{F}, \tilde{\mathcal{F}})$ consists of homotopy equivalence classes of elements $\Psi \in$ $\operatorname{Hom}_{\mathbb{C}[\mathcal{Z}]}\left(\mathrm{CE}_{\mathfrak{h}} \stackrel{\Delta}{\otimes} M, \mathrm{CE}_{\mathfrak{h}} \otimes \vec{\otimes} \tilde{M}\right)$ such that $\Psi \circ D_{\text {tot }}=\tilde{D}_{\text {tot }} \circ \Psi$ and $\Psi$ commutes with $U(\mathfrak{h})$-action on $\mathrm{CE}_{\mathfrak{h}} \otimes M$. Two maps $\Psi, \Psi^{\prime} \in \operatorname{Hom}(\mathcal{F}, \tilde{\mathcal{F}})$ are homotopy equivalent if there is

$$
h \in \operatorname{Hom}_{\mathbb{C}[\mathcal{Z}]}\left(\mathrm{CE}_{\mathfrak{h}} \stackrel{\Delta}{\otimes} M, \mathrm{CE}_{\mathfrak{h}} \stackrel{\Delta}{\otimes} \tilde{M}\right)
$$

such that $\Psi-\Psi^{\prime}=\tilde{D}_{\text {tot }} \circ h-h \circ D_{\text {tot }}$ and $h$ commutes with $U(h)$-action on $\mathrm{CE}_{\mathfrak{h}} \stackrel{\Delta}{\otimes} M$.

Given two $\mathfrak{h}$-equivariant matrix factorizations $\mathcal{F}=(M, D, \partial) \in \mathrm{MF}_{\mathfrak{h}}(\mathcal{Z}, F)$ and $\tilde{\mathcal{F}}=$ $(\tilde{M}, \tilde{D}, \tilde{\partial}) \in \operatorname{MF}_{\mathfrak{h}}(\mathcal{Z}, \tilde{F})$ we define $\mathcal{F} \otimes \tilde{\mathcal{F}} \in \operatorname{MF}_{\mathfrak{h}}(\mathcal{Z}, F+\tilde{F})$ as the equivariant matrix factorization $(M \otimes \tilde{M}, D+\tilde{D}, \partial+\tilde{\partial})$.

We define an embedding-related push-forward in the case when the subvariety $\mathcal{Z}_{0} \stackrel{j}{\hookrightarrow} \mathcal{Z}$ is the common zero of an ideal $I=\left(f_{1}, \ldots, f_{n}\right)$ such that the functions $f_{i} \in \mathbb{C}[\mathcal{Z}]$ form a regular sequence. We assume that the Lie algebra $\mathfrak{h}$ acts on $\mathcal{Z}$ and $I$ is $\mathfrak{h}$-invariant. In section 3 of OR18e we use technique similar to the proof of lemma 2.2.3 to show that there is a well-defined functor:

$$
j_{*}: \operatorname{MF}_{\mathfrak{h}}\left(\mathcal{Z}_{0},\left.W\right|_{\mathcal{Z}_{0}}\right) \longrightarrow \operatorname{MF}_{\mathfrak{h}}(\mathcal{Z}, W),
$$

for any $\mathfrak{h}$-invariant element $W \in \mathbb{C}[\mathcal{Z}]^{\mathfrak{h}}$.

For our construction of the convolution algebras we also need to define the equivariant push-forward along a projection. Suppose $\mathcal{Z}=\mathcal{X} \times \mathcal{Y}$, both $\mathcal{Z}$ and $\mathcal{X}$ have $\mathfrak{h}$-action and the projection $\pi: \mathcal{Z} \rightarrow \mathcal{X}$ is $\mathfrak{h}$-equivariant. Then for any $\mathfrak{h}$ invariant element $w \in \mathbb{C}[\mathcal{X}]^{\mathfrak{h}}$ there is a functor $\pi_{*}: \operatorname{MF}_{\mathfrak{h}}\left(\mathcal{Z}, \pi^{*}(w)\right) \rightarrow \operatorname{MF}_{\mathfrak{h}}(\mathcal{X}, w)$ which simply forgets the action of $\mathbb{C}[\mathcal{Y}]$.

Finally, let us discuss the quotient map. The complex $\mathrm{CE}_{\mathfrak{h}}$ is a resolution of the trivial $\mathfrak{h}$-module by free modules. Thus the correct derived version of taking $\mathfrak{h}$-invariant part of the matrix factorization $\mathcal{F}=(M, D, \partial) \in \operatorname{MF}_{\mathfrak{h}}(\mathcal{Z}, W), W \in \mathbb{C}[\mathcal{Z}]^{\mathfrak{h}}$ is

$$
\mathrm{CE}_{\mathfrak{h}}(\mathcal{F}):=\left(\mathrm{CE}_{\mathfrak{h}}(M), D+d_{c e}+\partial\right) \in \operatorname{MF}(\mathcal{Z} / H, W),
$$

where $\mathcal{Z} / H:=\operatorname{Spec}\left(\mathbb{C}[\mathcal{Z}]^{\mathfrak{h}}\right)$ and use the general definition for an $\mathfrak{h}$-module $V$ :

$$
\mathrm{CE}_{\mathfrak{h}}(V):=\operatorname{Hom}_{\mathfrak{h}}\left(\mathrm{CE}_{\mathfrak{h}}, \mathrm{CE}_{\mathfrak{h}} \stackrel{\Delta}{\otimes} V\right) .
$$




\section{BRAID GROUPS AND MATRIX FACTORIZATIONS}

In this section we explain a construction for an action of the finite and affine braid groups on the particular categories of the matrix factorizations from OR18e. First we explain a construction for the convolution algebra on our categories of matrix factorizations. Then we explain a categorization of the homomorphism from the affine braid group to the finite braid group from [OR18c].

3.1. Convolution product. Let us first motivate the definition of the space that host our categories of matrix factorizations. Somewhat abusing notations we introduce space $\sqrt{\mathcal{X}}=$ $\mathfrak{g l}_{n} \times \mathrm{GL}_{n} \times \mathfrak{n}$ where $\mathfrak{n}$ stands for the Lie algebra of strictly upper-triangular matrices. We omit the sub-index since the size of the matrices is clear from the context, we also use $G$ and $\mathfrak{g}$ for $\mathrm{GL}_{n}$ and $\mathfrak{g l}_{n}$ in this situation.

The space $\sqrt{\mathcal{X}}$ has the action of the group of upper-triangular matrices $B$ and $G$ :

$$
(h, b) \cdot(X, g, Y)=\left(\operatorname{Ad}_{h}(X), h g b, \operatorname{Ad}_{b}^{-1} Y\right), \quad(h, b) \in G \times B .
$$

The flag variety $\mathrm{Fl}$ is a quotient $G / B$ since every full flag can be moved into the standard flag by $G$-action and $B$ is the stabilizer group of the standard flag. The group $B$ acts on the tangent space to $\mathrm{Fl}$ at the point of standard flag and as $B$-module the tangent space is equal $\mathfrak{n}$. Thus the $B$-quotient of $\sqrt{\mathcal{X}}$ is naturally isomorphic to the cotangent bundle to the flag variety:

$$
\sqrt{\mathcal{X}} / B=\mathfrak{g} \times T^{*} \mathrm{Fl}
$$

Thus $G$-action on $\sqrt{\mathcal{X}}$ induces the $G$-action on $\mathfrak{g} \times T^{*} \mathrm{Fl}$.

The space $T^{*} \mathrm{Fl}$ is symplectic and the $G$-action respects preserves the symplectic form. Thus there is moment map $\mu: T^{*} \mathrm{Fl} \rightarrow \mathfrak{g}^{*}$. The trace identifies $\mathfrak{g}$ with $\mathfrak{g}^{*}$ and we can think of the moment map as $\mathfrak{g}$-linear $B$-invariant function:

$$
\mu: \sqrt{\mathcal{X}} \rightarrow \mathbb{C}, \quad \mu(X, g, Y)=\operatorname{Tr}\left(X \operatorname{Ad}_{g} Y\right) .
$$

Now we can define our main space where the convolution algebra dwells. The space $\sqrt{\mathcal{X}}$ has $B$-invariant projection to the first factor and our main space is the fibered product:

$$
\mathcal{X}:=\sqrt{\mathcal{X}} \times \mathfrak{g} \sqrt{\mathcal{X}}=\mathfrak{g} \times G \times \mathfrak{n} \times G \times \mathfrak{n} .
$$

The space $\mathcal{X}$ has a action of $G \times B^{2}$ that is induced from the $G \times B$ action on $\sqrt{\mathcal{X}}$, respectively the projections $p_{1}, p_{2}$ on two copies of $\sqrt{\mathcal{X}}$ are $G \times B^{2}$-equivariant. The group $B$ is a semi-direct product $B=T \ltimes U$ of the torus $T$ and the group of upper-triangular matrices $U$.

We define our main category to be:

$$
\mathrm{MF}_{n}:=\mathrm{MF}_{G \times B^{2}}(\mathcal{X}, W), \quad W=p_{1}^{*}(\mu)-p_{2}^{*}(\mu),
$$

where we require the weak $U^{2}$-equivariance and strong $G \times T^{2}$-equivariance in our category. The strong $G \times T^{2}$-equivariance implies that all differentials in the complexes are $G \times T^{2}$ invariant. We can combine strong $G \times T^{2}$-equivariance with the weak $U^{2}$-equivariance since the Chevalley-Eilenberg complex for $U^{2}$ is $G \times T^{2}$-invariant.

There is an action of $T_{s c}=\mathbb{C}^{*} \times \mathbb{C}^{*}=\mathbb{C}_{a}^{*} \times \mathbb{C}_{t}^{*}$ on the space $\sqrt{\mathcal{X}}$ and the induced action on $\mathcal{X}$ :

$$
(\lambda, \mu) \cdot(X, g, Y)=\left(\lambda^{2} \cdot X, g, \lambda^{-2} \mu^{2} Y\right) .
$$

The potential $W$ is not $T_{s c}$-invariant, it weight 2 with respect to the torus $\mathbb{C}_{t}^{*}$. We require the differentials in a curved complex from $\mathrm{MF}_{n}$ to have weight 1 with respect to $\mathbb{C}_{t}^{*}$ and it 
has weight 0 with respect to $\mathbb{C}_{a}^{*}$. To simplify notations we do not use any extra indices to indicate such $T_{s c}$-equivariance. We also use notation

$$
\mathbf{q}^{k} \mathbf{t}^{l} \cdot \mathcal{F}
$$

for the matrix factorization $\mathcal{F}$ with the $k$-twisted $\mathbb{C}_{a}^{*}$-action and $l$-twisted $\mathbb{C}_{t}^{*}$-action.

Since the space $\mathcal{X}$ has $B^{2}$-action we can also twist a matrix factorization $\mathcal{F}$ by a representation of this group. Given a characters $\chi_{l}$ and $\chi_{r}$ of the left and right factor in $B^{2}$, the twisted matrix factorization is denoted by

$$
\mathcal{F}\left\langle\chi_{l}, \chi_{r}\right\rangle \text {. }
$$

To define convolution product in category $\mathrm{MF}_{n}$ we introduce the convolution space $\mathcal{X}_{c o n}$ which is a fibered product:

$$
\mathcal{X}_{\text {con }}:=\sqrt{\mathcal{X}} \times_{\mathfrak{g}} \sqrt{\mathcal{X}} \times_{\mathfrak{g}} \sqrt{\mathcal{X}}=\mathfrak{g} \times(G \times n)^{2} .
$$

There are three $G \times B^{3}$-equivariant maps $\pi_{12}, \pi_{23}, \pi_{13}$ and the convolution product is defined by the predictable formula:

$$
\mathcal{F} \star \mathcal{G}:=\pi_{13 *}\left(\mathrm{CE}_{\mathfrak{n}(2)}\left(\pi_{12}^{*}(\mathcal{F}) \otimes \pi_{23}^{*}(\mathcal{G})\right)^{T^{(2)}}\right) .
$$

Since the projections $\pi_{i j}$ are smooth we can apply base change formula and the standard argument, that could be found in [CG97. One derives with the use of the base change that thus defined product is associative.

3.2. Knorrer reduction. We can apply the Knorrer periodicity discussed in section 2.3 to reduce the size of our working space $\mathcal{X}$. Indeed, the pair of space and potential:

$$
\overline{\mathcal{X}}=\mathfrak{b} \times G \times \mathfrak{n}, \quad \bar{W}(X, g, Y)=\operatorname{Tr}\left(X \operatorname{Ad}_{g}(Y)\right)
$$

is $B^{2}$-equivariant with respect to the action:

$$
\left(b_{1}, b_{2}\right) \cdot(X, g, Y)=\left(\operatorname{Ad}_{b_{1}} X, b_{1} g b_{2}, \operatorname{Ad}_{b_{2}}^{-1} Y\right) .
$$

Thus we can define the category of weakly $U^{2}$-equivariant and strongly $T^{2}$-equivariant matrix factorizations:

$$
\overline{\mathrm{MF}}_{n}:=\mathrm{MF}_{B^{2}}(\overline{\mathcal{X}}, \bar{W}) .
$$

To illustrate some of our methods we provide a proof for the equivalence in

Proposition 3.2.1. There is an equivalence of categories:

$$
\Psi: \overline{\mathrm{MF}}_{n} \rightarrow \mathrm{MF}_{n} .
$$

Proof. First we observe that the group $G$ acts freely on the space $\mathcal{X}$ hence we can take quotient by this group. The quotient can be implemented with help of the map:

$$
\mathcal{X} \stackrel{q}{\rightarrow} \mathcal{X}^{\circ}:=\mathfrak{g} \times \mathfrak{n} \times G \times \mathfrak{n}, \quad q\left(X, g_{1}, Y_{1}, g_{2}, Y_{2}\right)=\left(\operatorname{Ad}_{g_{1}}^{-1} X, Y_{1}, g_{1}^{-1} g_{2}, Y_{2}\right) .
$$

The potential $W^{\circ}\left(X, Y_{1}, g, Y_{2}\right)=\operatorname{Tr}\left(X\left(Y_{1}-\operatorname{Ad}_{g} Y_{2}\right)\right)$ is the pull-back $W_{0}=q^{*}(W)$ and the pull-back provides an equivalence $q^{*}: \mathrm{MF}_{n} \simeq \mathrm{MF}_{B^{2}}\left(\mathcal{X}^{0}, W_{0}\right)$.

To complete our proof we fix notations for the truncation of a square matrix $X$ :

$$
X=X_{+}+X_{--}, \quad X_{+} \in \mathfrak{n}, \quad X_{--}^{t} \in \mathfrak{b} .
$$


The potential $W^{\circ}$ can be written as a sum of $\bar{W}$ and a quadratic term and thus we can apply the Knorrer periodicity:

$$
\begin{aligned}
\operatorname{Tr}\left(X\left(Y_{1}-\operatorname{Ad}_{g} Y_{2}\right)\right) & =\operatorname{Tr}\left(\left(X_{+}+X_{--}\right)\left(Y_{1}-\operatorname{Ad}_{g} Y_{2}\right)\right)=-\operatorname{Tr}\left(X_{+}\left(\operatorname{Ad}_{g} Y_{2}\right)\right)+ \\
\operatorname{Tr}\left(X_{--}\left(Y_{1}-\operatorname{Ad}_{g} Y_{2}\right)\right) & =-\operatorname{Tr}\left(X_{+}\left(\operatorname{Ad}_{g} Y_{2}\right)\right)+\operatorname{Tr}\left(X_{--}\left(Y_{1}-\operatorname{Ad}_{g} Y_{2}\right)_{+}\right) .
\end{aligned}
$$

The entries of the matrices $X_{--}, Y_{1}-\left(\operatorname{Ad}_{g} Y_{2}\right)_{+}$are the coordinates in the direction transversal to the subspace $\overline{\mathcal{X}}$ with coordinates $X_{+}, g, Y_{2}$ and the Knorrer periodicity allows us to remove the quadratic term in the last formula.

It is explained in OR18e that the category $\overline{\mathrm{MF}}_{n}$ has a monoidal structure $\bar{\star}$ such that that the functor $\Psi$ sends it to the monoidal structure $\star$.

3.3. Braid groups. The affine braid group $\mathfrak{B r}_{n}^{a f f}$ is the group of braids whose strands may also wrap around a 'flag pole'. The group is generated by the standard generators $\sigma_{i}$, $i=1, \ldots, n-1$ and a braid $\Delta_{n}$ that wraps the last stand of the braid around the flag pole:

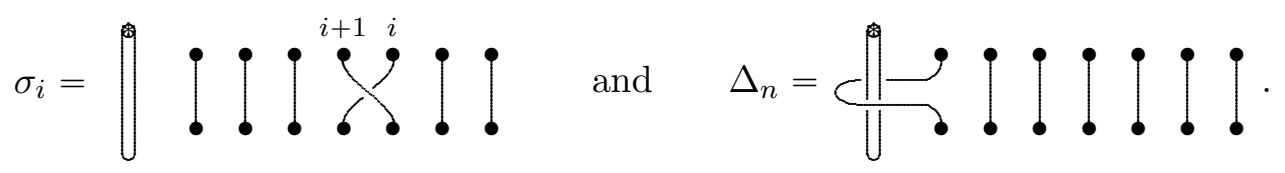

The defining relations for this generators are

$$
\begin{gathered}
\sigma_{n-1} \cdot \Delta_{n} \cdot \sigma_{n-1} \cdot \Delta_{n}=\Delta_{n} \cdot \sigma_{n-1} \cdot \Delta_{n} \cdot \sigma_{n-1}, \\
\sigma_{i} \cdot \Delta_{n}=\Delta_{n} \cdot \sigma_{i}, \quad i<n-1, \\
\sigma_{i} \cdot \sigma_{i+1} \cdot \sigma_{i}=\sigma_{i+1} \cdot \sigma_{i} \cdot \sigma_{i+1}, \quad i=1, \ldots, n-2, \\
\sigma_{i} \cdot \sigma_{j}=\sigma_{j} \cdot \sigma_{i}, \quad|i-j|>1 .
\end{gathered}
$$

The mutually commuting Bernstein-Lusztig (BL) elements $\Delta_{i} \in \mathfrak{B r}_{n}^{a f f}$ are defined as follows:

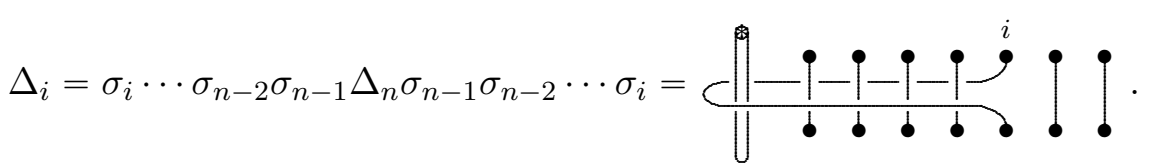

The finite braid group $\mathfrak{B r}_{n}$ is the subgroup of the affine braid group with the generators $\sigma_{i}, i=1, \ldots, n-1$. Other words, we do not allow the braids to go around the pole.

There is a natural homomorphism $\mathfrak{f g t}: \mathfrak{B r}_{n}^{a f f} \rightarrow \mathfrak{B r}_{n}$, geometrically it is defined by removing the flag pole. In particular we have:

$$
\mathfrak{f} \mathfrak{g t}\left(\Delta_{n}\right)=1, \quad \mathfrak{f} \mathfrak{g t}\left(\Delta_{i}\right)=\delta_{i}, \quad i=1, \ldots, n-1 .
$$

The inclusion discussed above $i_{f i n}: \mathfrak{B r}_{n} \rightarrow \mathfrak{B r}_{n}^{a f f}$ is a section of the flag forgetting map: $\mathfrak{f} \mathfrak{r g} \circ i_{\text {fin }}=1$. 
3.4. Braid action. In this section we outline a construction of the homomorphisms from the (affine) braid group to our convolution algebras of matrix factorizations. For a geometric counter-part of the map $\mathfrak{g}$ we need to introduce stable versions of our categories of matrix factorizations.

Let us define the stable locus $\overline{\mathcal{X}}^{s t, \bullet} \subset \overline{\mathcal{X}} \times V$ to be set of quadruples $(X, g, Y, v)$ that satisfy a open condition:

$$
\mathbb{C}\left\langle\left(\operatorname{Ad}_{g}^{-1} X\right)_{+}, Y\right\rangle v=V .
$$

There is a natural projection $\pi_{V}: \overline{\mathcal{X}} \times V \rightarrow \overline{\mathcal{X}}$ and there is an open embedding map $j_{s t}: \overline{\mathcal{X}}^{s t} \rightarrow \overline{\mathcal{X}}$ where $\overline{\mathcal{X}}^{s t}=\pi_{V}\left(\overline{\mathcal{X}}^{\bullet}, s t\right)$. This map induces the pull-back map:

$$
j_{s t}^{*}: \overline{\mathrm{MF}}_{n} \rightarrow \overline{\mathrm{MF}}_{n}^{s t}:=\mathrm{MF}_{B^{2}}\left(\overline{\mathcal{X}}^{s t}, \bar{W}\right)
$$

It is shown in [OR18e, Lemma 13.3] that the category $\overline{\mathrm{MF}}_{n}$ has a natural structure of convolution algebra. The main results of the papers OR18e, OR18c play the crucial role in the construction of the knot invariant in the next section.

Theorem 3.4.1. There are homomorphisms of algebras:

$$
\Phi: \mathfrak{B r}_{n} \rightarrow\left(\overline{\mathrm{MF}}_{n}^{s t}, \bar{\star}\right), \quad \Phi^{\text {aff }}: \mathfrak{B r}_{n}^{a f f} \rightarrow\left(\overline{\mathrm{MF}}_{n}, \bar{\star}\right) .
$$

Moreover, the pull-back $j_{\text {st }}^{*}$ is the homomorphism of the convolution algebras and

$$
j_{s t}^{*} \circ \Phi^{a f f}=\Phi \circ \text { fgt. }
$$

The fact that the pull-back morphism is an algebra homomorphism relies on the following shrinking lemma, for a proof see lemma 12.3 in [OR18e.

Lemma 3.4.2. Suppose $X$ is a quasi-affine variety and $\mathcal{F}=(M, D) \in \operatorname{MF}^{s c}(X, W), W \in$ $\mathbb{C}[X]$. The elements of $\mathbb{C}[X]$ act on $\operatorname{MF}(X, W)$ by multiplication. Let us assume that the elements of the ideal $I=\left(f_{1}, \ldots, f_{m}\right) \subset \mathbb{C}[X]$ act by zero-homotopic endomorphisms on $\mathcal{F}$ and $Z^{\prime} \subset X$ is the zero locus of $I$. Let $Z \subset X$ be a subvariety defined by $J=\left(g_{1}, \ldots, g_{n}\right)$ such that $Z \cap Z^{\prime}=\varnothing$. Then $\mathcal{F}$ is homotopic to $\left.\mathcal{F}\right|_{X \backslash Z}$ as matrix factorization over $\mathbb{C}[X]$.

Essentially the lemma says that we can shrink our ambient space to any open neighborhood of the critical locus of the potential and such operation does not change the corresponding category of matrix factorizations.

Let us also remark that there is another construction for the affine braid group action on the similar category of matrix factorizations in [AK15b] but the precise relation between our construction and result of this paper is known to the author.

\section{KNOT INVARIANTS}

4.1. Geometric trace operator. Let $\mathfrak{b}_{n}, \mathfrak{n}_{n}$ be Lie algebras of the group of upper, respectively strictly-upper triangular $n \times n$ matrices. The free nested Hilbert scheme FHilb ${ }_{n}^{\text {free }}$ is a $B \times \mathbb{C}^{*}$-quotient of the sublocus $\widetilde{\text { FHilb }}_{n}^{\text {free }} \subset \mathfrak{b}_{n} \times \mathfrak{n}_{n} \times V_{n}$ of the cyclic triples

$$
\widetilde{\text { FHilb }}_{n}^{\text {free }}=\left\{(X, Y, v) \mid \mathbb{C}\langle X, Y\rangle v=V_{n}\right\},
$$

here $V_{n}=\mathbb{C}^{n}$. The usual nested Hilbert scheme FHilb ${ }_{n}$ is the subvariety of FHilb free, it is defined by the condition that $X, Y$ commute.

Remark 4.1.1. There is a bit of confusion in the notations, what we denote here by FHilb ${ }_{n}$ is denoted in OR18e] by Hilb $1, n$ and by $\mathrm{FHilb}_{n}(\mathbb{C})$ in $[\mathrm{GRN16}$. 
The torus $T_{s c}=\mathbb{C}^{*} \times \mathbb{C}^{*}$ acts on FHilb ${ }_{n}^{\text {rree }}$ by scaling the matrices. We denote by $D_{T_{s c}}^{\text {per }}\left(\right.$ FHilb $\left._{n}^{\text {free }}\right)$ a derived category of the two-periodic complexes of the $T_{s c}$-equivariant quasicoherent sheaves on $\mathrm{FHilb}_{n}^{\text {free }}$. Let us also denote by $\mathcal{B}^{\vee}$ the descent of the trivial vector

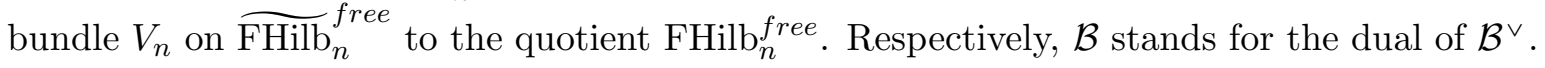
Below we construct for every $\beta \in \mathfrak{B r}_{n}$ an element

$$
\mathbb{S}_{\beta} \in D_{T_{s c}}^{\text {per }}\left(\mathrm{FHilb}_{n}^{\text {free }}\right)
$$

such that space of hyper-cohomology of the complex:

$$
\mathbb{H}^{k}\left(\mathbb{S}_{\beta}\right):=\mathbb{H}\left(\mathbb{S}_{\beta} \otimes \Lambda^{k} \mathcal{B}\right)
$$

defines an isotopy invariant.

Theorem 4.1.2. OR18e For any $\beta \in \mathfrak{B r}_{n}$ the doubly graded space

$$
H^{k}(\beta):=\mathbb{H}^{(k+\operatorname{writh}(\beta)-n-1) / 2}\left(\mathbb{S}_{\beta}\right)
$$

is an isotopy invariant of the braid closure $L(\beta)$.

The variety $\widetilde{\mathrm{FHilb}}_{n}^{\text {free }}$ embeds inside $\overline{\mathcal{X}}$ via a map $j_{e}:(X, Y, v) \rightarrow(X, e, Y, v)$. The diagonal copy $B=B_{\Delta} \hookrightarrow B^{2}$ respects the embedding $j_{e}$ and since $j_{e}^{*}(\bar{W})=0$, we obtain a functor:

$$
j_{e}^{*}: \mathrm{MF}_{B_{n}^{2}}\left(\overline{\mathcal{X}}^{s t}, \bar{W}\right)={\overline{\mathrm{MF}_{n}^{s t}}}^{s t} \mathrm{MF}_{B_{\Delta}}\left(\widetilde{\mathrm{FHilb}}_{n}^{\text {free }}, 0\right) .
$$

Respectively, we get a geometric version of "closure of the braid" map:

$$
\mathbb{L}: \mathrm{MF}_{B_{n}^{2}}\left(\overline{\mathcal{X}}^{s t}, \bar{W}\right)=\overline{\mathrm{MF}}_{n}^{s t} \rightarrow D_{T_{s c}}^{\text {per }}\left(\text { FHilb }_{n}^{\text {free }}\right) .
$$

The main result of [OR18e] could be restated in more geometric term via geometric trace map:

$$
\mathcal{T} r: \mathfrak{B r}_{n} \rightarrow D_{T_{s c}}^{\text {per }}\left(\mathrm{FHilb}_{n}^{\text {free }}\right), \quad \mathcal{T} r(\beta):=\oplus_{k} \mathbb{L}\left(\Phi(\beta) \otimes \Lambda^{k} \mathcal{B}\right)
$$

The above mentioned complex $\mathbb{S}_{\beta}$ is the complex $\mathbb{L}(\Phi(\beta))$. The differentials in the complex $\mathbb{S}_{\beta}$ are of degree $t$ thus the differentials are invariant with respect to the anti-diagonal torus $T_{a}$. Hence the forgetful functor $\chi: D_{T_{s c}}^{\text {per }}\left(\mathrm{FHilb}^{\text {free }}\right) \rightarrow D_{T_{a}}^{\text {per }}$ (FHilb ${ }^{\text {free }}$ ) could be composed with $K$-theory functor $\mathrm{K}: D_{T_{a}}^{\text {per }}\left(\right.$ FHilb $\left.^{\text {free }}\right) \rightarrow K_{T_{a}}\left(\right.$ FHilb $\left.^{\text {free }}\right)$. The composite functor $\mathrm{K} \circ \chi$ is closely related to decategorification and the classical Ocneanu-Jones trace, we discuss the Ocneaunu-Jones trace $\operatorname{Tr}^{O J}$ and related theorem of Markov in the next subsection.

Theorem 4.1.3. OR18e The composition $\mathbb{H} \circ \mathcal{T} r: \mathfrak{B r}_{n} \rightarrow D_{T_{s c}}^{p e r}(p t)$ categorifies the JonesOceanu trace:

$$
\operatorname{Tr}^{O J}(\beta)=\operatorname{dim}_{a, q} \mathrm{~K} \circ \chi \circ \mathbb{H} \circ \mathcal{T} r(\beta),
$$

where the q-grading comes $T_{a}$-action and a-grading is from the exterior powers of $\mathcal{B}$

4.2. OJ trace and Markov theorem. As we discussed before every link $L$ in $\mathbb{R}^{3}$ is isotopic to the closure of a braid $L=\mathrm{L}(\beta), \beta \in \mathfrak{B r}_{n}$. On the other hand it is clear that such a presentation is not unique. Markov theorem describes the non-uniqueness explicitly and thus provides an algebraic description of the set $\mathfrak{L}$ of the isotopy equivalence classes of the links.

Theorem 4.2.1. The closure operation $\mathrm{L}$ identifies the set $\mathfrak{L}$ of isotopy class of links in $S^{3}$ and the set of equivalence classes:

$$
\bigcup_{n} \mathfrak{B r}_{n} / \sim
$$


where the equivalence relation is generated by the elementary equivalences:

$$
\begin{gathered}
\alpha \cdot \beta \sim \beta \cdot \alpha, \quad \alpha, \beta \in \mathfrak{B r}_{n} \\
\mathfrak{B r}_{n+1} \ni \alpha \cdot \sigma^{ \pm 1} \sim \alpha, \quad \alpha \in \mathfrak{B r}_{n} .
\end{gathered}
$$

If we have homomorphism $\operatorname{Tr}$ from the braid group to some field $F$ that respects the relations (4.2) then the value $\operatorname{Tr}(\beta) \in F$ is an isotopy invariant of the closure $\mathrm{L}(\beta)$. In practice it is hard to classify such homomorphisms, however the great discovery of Ocneanu and Jones is that one can classify such homomorphisms if we pass to a quotient $H_{n}$ of the braid group.

The Hecke algebra $H_{n}$ is generated by $g_{i}, i=1, \ldots, n-1$ modulo relations:

$$
\begin{gathered}
g_{i} g_{i+1} g_{i}=g_{i+1} g_{i} g_{i+1}, \quad i=1, \ldots, n-2, \\
g_{i}-g_{i}^{-1}=q-q^{-1}, \quad i=1, \ldots, n-1 .
\end{gathered}
$$

There is a natural algebra homomorphism $\pi: \mathfrak{B r}_{n} \rightarrow H_{n}, \sigma_{i} \mapsto g_{i}$. It is shown in Jon87] that there is a unique homomorphism $\operatorname{Tr}^{O J}: \bigcup_{n} H_{n} \rightarrow \mathbb{Q}(a, q)$ that satisfies relations (4.2) and normalizing relation

$$
\operatorname{Tr}^{O J}(\mathbf{1})=\frac{a-a^{-1}}{q-q^{-1}}
$$

The corresponding invariant is $\operatorname{Tr}^{O J}(\beta) \in \mathbb{Q}(a, q)$ is also known as HOMFLYPT invariant, $\operatorname{HOMFLYPT}(\beta)$.

Thus the formula (1.1) from the introduction and theorem 4.1.3 state that there is a specialization of the graded dimension of $\operatorname{HHH}(\beta)$ that becomes a HOMFLYPT invariant. Let us recall that the $\operatorname{space} \operatorname{HHH}(\beta)$ up to some elementary grading shift is equal:

$$
\mathbb{H}^{*}\left(\Phi(\beta) \otimes \Lambda^{\bullet} \mathcal{B}\right)^{B}
$$

This space naturally has four gradings: $*, \bullet$ and $T_{s c}$-grading. However, only three of these gradings are invariant with respect to the Markov moves: $*$ is not preserved by the moves. The first grading is $\bullet$, we call it $a$-grading, since it is responsible for the $a$-variable in the HOMFLYPT polynomial specializations. The other two gradings come from $T_{s c}$-action:

$$
\operatorname{deg}\left(X_{i j}\right)=q^{2}, \quad \operatorname{deg}\left(Y_{i j}\right)=q^{-2} t^{-2} .
$$

To specialize to the HOMFLYPT polynomial we need to set $t=-1$ or more geometrically, we need to restrict torus $T_{s c}$-action on the $\operatorname{space} \operatorname{HHH}(\beta)$ to the action of the anti-diagonal torus, we denote the specialized category by $\overline{\mathrm{MF}}_{n}^{s t, a}$. To be more precise, the category $\mathrm{MF}_{n}^{s t, a}$ is a category of matrix factorizations on $\overline{\mathcal{X}}^{s t}$ with the potential $\bar{W}$ which are $B_{n}^{2}$-weakly equivariant, $G \times T^{2} \times T_{a}$-strongly equivariant.

As we mentioned before this torus is special because under this specialization the differentials in the curved complexes from $\overline{\mathrm{MF}}_{n}^{s t}$ become torus invariant, hence there is a well-defined functor:

$$
\mathrm{K}: \quad \overline{\mathrm{MF}}_{n}^{s t, a} \rightarrow \mathrm{K}_{T_{a}}\left(\mathrm{MF}_{n}^{s t, a}\right) .
$$

This K-theory functor turns the homotopy equivalence (6.1) into the relation:

$$
\left[\mathcal{C}_{+}\right]=q^{-1}\left(\left[\mathcal{C}_{\|}\right]-\left[\mathcal{C} \cdot\left\langle-\chi_{1},-\chi_{1}\right\rangle\right]\right)
$$

Thus the combination of the relations (1.2) and (6.3), (6.4) imply the quadratic relation in the Hecke algebra. 
The Markov move relations (4.2) hold for the invariant $\operatorname{HHH}(\beta)$ and in the section 7 we discuss the main idea of our proof of the Markov moves for HHH. We need some details of the braid group action construction for the Markov move argument, therefore we outline the construction in the next section.

\section{Geometric realization of the affine braid group}

5.1. Induction functors. The standard parabolic subgroup $P_{k}$ has Lie algebra generated by $\mathfrak{b}$ and $E_{i+1, i}, i \neq k$. Let us define space $\overline{\mathcal{X}}\left(P_{k}\right):=\mathfrak{b} \times P_{k} \times \mathfrak{n}$ and let us also use notation $\overline{\mathcal{X}}\left(\mathrm{GL}_{n}\right)$ for $\overline{\mathcal{X}}\left(\mathrm{GL}_{n}\right)$. There is a natural embedding $\bar{i}_{k}: \overline{\mathcal{X}}\left(P_{k}\right) \rightarrow \overline{\mathcal{X}}$ and a natural projection $\bar{p}_{k}: \overline{\mathcal{X}}\left(P_{k}\right) \rightarrow \overline{\mathcal{X}}\left(\mathrm{GL}_{k}\right) \times \overline{\mathcal{X}}\left(\mathrm{GL}_{n-k}\right)$. The space $\overline{\mathcal{X}}\left(\mathrm{GL}_{k}\right) \times \overline{\mathcal{X}}\left(\mathrm{GL}_{n-k}\right)$ is equipped with a $B_{k}^{2} \times B_{n-k}^{2}$-invariant potential $\bar{W}^{(1)}+\bar{W}^{(2)}$ which is a sum of pull-backs of the potentials $W$ along the projection on the first and the second factors. Moreover, we have:

$$
\bar{i}_{k}^{*}(\bar{W})=\bar{p}_{k}^{*}\left(\bar{W}^{(1)}+\bar{W}^{(2)}\right) .
$$

Since the embedding $\bar{i}_{k}$ satisfies the conditions for existence of the push-forward and the relation (5.1) between the potentials holds, we can define the induction functor:

$$
\overline{\operatorname{ind}}_{k}:=\bar{i}_{k *} \circ \bar{p}_{k}^{*}: \mathrm{MF}_{B_{k}^{2}}\left(\overline{\mathcal{X}}\left(\mathrm{GL}_{k}\right), \bar{W}\right) \times \mathrm{MF}_{B_{n-k}^{2}}\left(\overline{\mathcal{X}}\left(\mathrm{GL}_{n-k}\right), \bar{W}\right) \rightarrow \mathrm{MF}_{B_{n}^{2}}\left(\overline{\mathcal{X}}\left(\mathrm{GL}_{n}\right), \bar{W}\right)
$$

Similarly we define space $\overline{\mathcal{X}}_{f r}\left(P_{k}\right) \subset \mathfrak{b} \times P_{k} \times \mathfrak{n} \times V$ as an open subset defined by the stability condition (3.1). The last space has a natural projection map

$$
\bar{p}_{k}: \overline{\mathcal{X}}_{f r}\left(P_{k}\right) \rightarrow \overline{\mathcal{X}}\left(\mathrm{GL}_{k}\right) \times \overline{\mathcal{X}}_{f r}\left(\mathrm{GL}_{n-k}\right)
$$

and the embedding $\bar{i}_{k}: \overline{\mathcal{X}}_{f r}\left(P_{k}\right) \rightarrow \overline{\mathcal{X}}_{f r}\left(\mathrm{GL}_{n}\right)$ and we can define the induction functor:

$$
\overline{\operatorname{ind}}_{k}:=\bar{i}_{k *} \circ \bar{p}_{k}^{*}: \operatorname{MF}_{B_{k}^{2}}\left(\overline{\mathcal{X}}\left(\mathrm{GL}_{k}\right), \bar{W}\right) \times \operatorname{MF}_{B_{n-k}^{2}}\left(\overline{\mathcal{X}}_{f r}\left(\mathrm{GL}_{n-k}\right), \bar{W}\right) \rightarrow \mathrm{MF}_{B_{n}^{2}}\left(\overline{\mathcal{X}}_{f r}\left(\mathrm{GL}_{n}\right), \bar{W}\right)
$$

It is shown in section 6 (proposition 6.2 ) of OR18e that the functor $\overline{\text { ind }_{k}}$ is the homomorphism of the convolution algebras:

$$
\overline{\operatorname{ind}}_{k}\left(\mathcal{F}_{1} \bowtie \mathcal{F}_{2}\right) \overline{\operatorname{ind}}_{k}\left(\mathcal{G}_{1} \bowtie \mathcal{G}_{2}\right)=\overline{\operatorname{ind}}_{k}\left(\mathcal{F}_{1} \star \mathcal{G}_{2} \bowtie \mathcal{F}_{2} \star \mathcal{G}_{2}\right) .
$$

To define the non-reduced version of the induction functors one needs to introduce the space $\mathcal{X}^{\circ}\left(\mathrm{GL}_{n}\right)=\mathfrak{g} \times \mathrm{GL}_{n} \times \mathfrak{n} \times \mathfrak{n}$ which is the slice to $\mathrm{GL}_{n}$-action on the space $\mathcal{X}$. In particular, the potential $W$ on this slice becomes:

$$
W\left(X, g, Y_{1}, Y_{2}\right)=\operatorname{Tr}\left(X\left(Y_{1}-\operatorname{Ad}_{g}\left(Y_{2}\right)\right)\right) .
$$

Similarly to the case of the reduced space, one can define the space $\mathcal{X}^{\circ}\left(P_{k}\right):=\mathfrak{g} \times P_{k} \times \mathfrak{n} \times \mathfrak{n}$ to be the locus of and the corresponding maps $i_{k}: \mathcal{X}^{\circ}\left(P_{k}\right) \rightarrow \mathcal{X}^{\circ}\left(\mathrm{GL}_{n}\right), p_{k}: \mathcal{X}^{\circ}\left(P_{k}\right) \rightarrow$ $\mathcal{X}^{\circ}\left(\mathrm{GL}_{k}\right) \times \mathcal{X}^{\circ}\left(\mathrm{GL}_{n-k}\right)$. Thus we get a version of the induction functor for non-reduced spaces:

$$
\operatorname{ind}_{k}:=i_{k *} \circ p_{k}^{*}: \mathrm{MF}_{B_{k}^{2}}\left(\mathcal{X}\left(\mathrm{GL}_{k}\right), W\right) \times \mathrm{MF}_{B_{n-k}^{2}}\left(\mathcal{X}\left(\mathrm{GL}_{n-k}\right), W\right) \rightarrow \mathrm{MF}_{B_{n}^{2}}\left(\mathcal{X}\left(\mathrm{GL}_{n}\right), W\right)
$$

It is shown in proposition 6.1 of OR18e that the Knörrer functor is compatible with the induction functor:

$$
\operatorname{ind}_{k} \circ\left(\Phi_{k} \times \Phi_{n-k}\right)=\Phi_{n} \circ \operatorname{ind}_{k}
$$


5.2. Generators of the finite braid group action. Let us define $B^{2}$-equivariant embed$\operatorname{ding} i: \overline{\mathcal{X}}\left(B_{n}\right) \rightarrow \overline{\mathcal{X}}, \overline{\mathcal{X}}(B):=\mathfrak{b} \times B \times \mathfrak{n}$. The pull-back of $\bar{W}$ along the map $i$ vanishes and the embedding $i$ satisfies the conditions for existence of the push-forward $i_{*}: \operatorname{MF}_{B^{2}}\left(\overline{\mathcal{X}}\left(B_{n}\right), 0\right) \rightarrow$ $\mathrm{MF}_{B^{2}}\left(\overline{\mathcal{X}}\left(\mathrm{GL}_{n}\right), \bar{W}\right)$. We denote by $\mathbb{C}\left[\overline{\mathcal{X}}\left(B_{n}\right)\right] \in \mathrm{MF}_{B^{2}}\left(\overline{\mathcal{X}}\left(B_{n}\right), 0\right)$ the matrix factorization with zero differential that is concentrated only in even homological degree. As it is shown in proposition 7.1 of OR18e the push-forward

$$
\overline{\mathbb{1}}_{n}:=i_{*}\left(\underline{\mathbb{C}\left[\overline{\mathcal{X}}\left(B_{n}\right)\right]}\right)
$$

is the unit in the convolution algebra. Similarly, $\mathbb{1}_{n}:=\Phi\left(\overline{\mathbb{1}}_{n}\right)$ is also a unit in non-reduced case.

Let us first discuss the case of the braids on two strands. The key to construction of the braid group action in OR18e is the following factorization in the case $n=2$ :

$$
\bar{W}(X, g, Y)=y_{12}\left(2 g_{11} x_{11}+g_{21} x_{12}\right) g_{21} / \operatorname{det},
$$

where $\operatorname{det}=\operatorname{det}(g)$ and

$$
g=\left[\begin{array}{ll}
g_{11} & g_{12} \\
g_{21} & g_{22}
\end{array}\right], \quad X=\left[\begin{array}{cc}
x_{11} & x_{12} \\
0 & x_{22}
\end{array}\right], \quad Y=\left[\begin{array}{cc}
0 & y_{12} \\
0 & 0
\end{array}\right]
$$

Thus we can define the following strongly equivariant Koszul matrix factorization:

$$
\begin{aligned}
& \overline{\mathcal{C}}_{+}:=(\mathbb{C}[\overline{\mathcal{X}}] \otimes \Lambda\langle\theta\rangle, D) \in \mathrm{MF}_{B^{2}}^{s \operatorname{tr}}(\overline{\mathcal{X}}, \bar{W}), \\
& D=\frac{g_{12} y_{12}}{\operatorname{det}} \theta+\left(g_{11}\left(x_{11}-x_{22}\right)+g_{21} x_{12}\right) \frac{\partial}{\partial \theta},
\end{aligned}
$$

where $\Lambda\langle\theta\rangle$ is the exterior algebra with one generator.

This matrix factorization corresponds to the positive elementary braid on two strands. Using the induction functor we can extend the previous definition to the case of the arbitrary number of strands. For that we introduce an insertion functor:

$$
\begin{aligned}
& \overline{\operatorname{Ind}}_{k, k+1}: \mathrm{MF}_{B_{2}^{2}}\left(\overline{\mathcal{X}}\left(\mathrm{GL}_{2}\right), \bar{W}\right) \rightarrow \mathrm{MF}_{B_{n}^{2}}\left(\overline{\mathcal{X}}\left(\mathrm{GL}_{n}\right), \bar{W}\right) \\
& \overline{\operatorname{Ind}}_{k, k+1}(\mathcal{F}):=\overline{\operatorname{ind}}_{k+1}\left(\overline{\operatorname{ind}}_{k-1}\left(\overline{\mathbb{1}}_{k-1} \times \mathcal{F}\right) \times \overline{\mathbb{1}}_{n-k-1}\right),
\end{aligned}
$$

and similarly we define non-reduced insertion functor

$$
\operatorname{Ind}_{k, k+1}: \mathrm{MF}_{B_{2}^{2}}\left(\mathcal{X}\left(G_{2}\right), W\right) \rightarrow \mathrm{MF}_{B_{n}^{2}}\left(\mathcal{X}\left(G_{n}\right), W\right) .
$$

Thus we define the generators of the braid group as follows:

$$
\overline{\mathcal{C}}_{+}^{(k)}:=\overline{\operatorname{Ind}}_{k, k+1}\left(\overline{\operatorname{ind}}_{k-1}\left(\overline{\mathcal{C}}_{+}\right)\right), \quad \overline{\mathcal{C}}_{+}^{(k)}:=\operatorname{Ind}_{k, k+1}\left(\operatorname{ind}_{k-1}\left(\mathcal{C}_{+}\right)\right) .
$$

The section 11 of OR18e is devoted to the proof of the braid relations between these elements:

$$
\begin{gathered}
\overline{\mathcal{C}}_{+}^{(k+1)} \bar{\star}^{(k)} \overline{\mathcal{C}}_{+}^{(k)} \overline{\mathcal{\mathcal { C }}_{+}^{(k+1)}}=\overline{\mathcal{C}}_{+}^{(k)} \overline{\mathcal{C}}_{+} \overline{\mathcal{C}}_{+}^{(k+1)} \bar{\star} \overline{\mathcal{C}}_{+}^{(k)}, \\
\mathcal{C}_{+}^{(k+1)} \star \mathcal{C}_{+}^{(k)} \star \mathcal{C}_{+}^{(k+1)}=\mathcal{C}_{+}^{(k)} \star \mathcal{C}_{+}^{(k+1)} \star \mathcal{C}_{+}^{(k)}
\end{gathered}
$$

Let us now discuss the inversion of the elementary braid. In view of inductive definition of the braid group action, it is sufficient to understand the inversion in the case $n=2$.

Thus we define:

$$
\mathcal{C}_{-}:=\mathcal{C}_{+}\left\langle-\chi_{1}, \chi_{2}\right\rangle \in \mathrm{MF}_{B^{2}}\left(\mathcal{X}\left(\mathrm{GL}_{2}\right), W\right)
$$


where $\chi_{1}, \chi_{2}$ are the standard generators of the character group of $\mathbb{C}^{*} \times \mathbb{C}^{*}=T \subset B_{2}$. The definition of $\overline{\mathcal{C}}_{-}$is similar. It could be shown that the definition of $\mathcal{C}_{-}$is actually symmetric with respect to the left-right twisting:

$$
\mathcal{C}_{-}=\mathcal{C}_{+}\left\langle\chi_{2},-\chi_{1}\right\rangle
$$

Theorem 5.2.1. We have:

$$
\mathcal{C}_{+} \star \mathcal{C}_{-}=\mathbb{1}_{2}
$$

\section{SAMPlE COMPUTATiON}

In this section we would like to show an example of the convolution algebra computations. But before I would expand a little bit our discussion of the basic matrix factorizations in the case of $n=2$.

6.1. Basic matrix factorizations of rank 2. We have shown in the previous section that the potential $\bar{W}$ is a product of three factors and we used this fact to define the matrix factorization $\overline{\mathcal{C}}_{+}$. However, it is clear that there are two more natural matrix factorizations for this potential:

$$
\begin{gathered}
\overline{\mathcal{C}}_{\|}:=\left(\mathbb{C}[\overline{\mathcal{X}}] \otimes \Lambda\langle\theta\rangle, D_{\|}, 0,0\right), \quad \overline{\mathcal{C}} \bullet:=\left(\mathbb{C}[\overline{\mathcal{X}}] \otimes \Lambda\langle\theta\rangle, D_{\bullet}, 0,0\right) \in \mathrm{MF}_{B^{2}}(\overline{\mathcal{X}}, \bar{W}), \\
D_{\|}=\frac{g_{21}}{\operatorname{det}} \theta+y_{12} \tilde{x}_{0} \frac{\partial}{\partial \theta}, \quad D_{\bullet}=\frac{g_{21}}{\operatorname{det}} \tilde{x}_{0} \theta+y_{12} \frac{\partial}{\partial \theta}, \quad \tilde{x}_{0}=g_{11}\left(x_{11}-x_{22}\right)+g_{21} x_{12} .
\end{gathered}
$$

One of the matrix factorizations is actually a cone of the morphism between the other two:

$$
\left[\overline{\mathcal{C}}_{\|} \stackrel{\phi}{\rightarrow} \overline{\mathcal{C}}_{\bullet}\left\langle-\chi_{1},-\chi_{1}\right\rangle\right] \sim \mathbf{q} \mathbf{t} \cdot \overline{\mathcal{C}}_{+}
$$

with map $\phi$ defined by

$$
\begin{array}{rr}
\mathbf{t}^{-1} \cdot R\left\langle\chi_{2},-\chi_{1}\right\rangle \stackrel{1}{\longrightarrow} & R\left\langle\chi_{2},-\chi_{1}\right\rangle \\
y_{12} \tilde{x}_{0} \uparrow \mid g_{21} & y_{12} \uparrow \mid \downarrow g_{21} \tilde{x}_{0} \\
R & R\left\langle 0,-\chi_{1}\right\rangle,
\end{array}
$$

where $R=\mathbb{C}[\overline{\mathcal{X}}]$. This relation is crucial for our discussion of the connection with the Oceanus-Jones traces

6.2. Details on the convolution product. The convolution product inside the category $\mathrm{MF}_{B^{2}}(\overline{\mathcal{X}}, \bar{W})$ is a bit tricky to define and we refer reader to our paper OR18e] where the convolution product is constructed and used for the computations for $n=3$. On the other hand the space $\mathcal{X}$ is bigger than the space $\overline{\mathcal{X}}$ but the construction of the convolution is more straightforward. The space $\mathcal{X}^{\circ}:=\mathcal{X} / \mathrm{GL}_{n}=\mathfrak{g} \times \mathfrak{n} \times \mathrm{GL}_{n} \times \mathfrak{n}$ is intermediate between these two spaces and we choose to work with this slightly bigger space to make our exposition simpler.

The space $\mathcal{X}^{\circ}$ and the relevant potential $W^{\circ}$ appeared already in the proof of the proposition 3.2.1. Let us spell out the definition of the convolution structure for the elements $\mathcal{F}, \mathcal{G} \in \operatorname{MF}_{B^{2}}\left(\mathcal{X}^{\circ}, W^{\circ}\right):$

$$
\mathcal{F} \star \mathcal{G}:=\pi_{13 *}^{\circ}\left(\mathrm{CE}\left(\pi_{12}^{\circ *}(\mathcal{F}) \otimes \pi_{23}^{\circ *}(\mathcal{G})\right)\right)
$$

where we used the convolution space $\mathcal{X}_{c n v}^{\circ}:=\mathfrak{g} \times \mathfrak{n} \times \mathrm{GL}_{n} \times \mathfrak{n} \times \mathrm{GL}_{n} \times \mathfrak{n}$ and the $B^{3}$-equivariant maps are

$$
\begin{gathered}
\pi_{12}^{\circ}\left(X, Y_{1}, g_{12}, Y_{2}, g_{23}, Y_{3}\right)=\left(X, Y_{1}, g_{12}, Y_{2}\right), \quad \pi_{23}^{\circ}\left(X, Y_{1}, g_{12}, Y_{2}, g_{23}, Y_{3}\right)=\left(\operatorname{Ad}_{g_{12}} X, Y_{2}, g_{23}, Y_{3}\right), \\
\pi_{13}^{\circ}\left(X, Y_{1}, g_{12}, Y_{2}, g_{23}, Y_{3}\right)=\left(X, Y_{1}, g_{12} g_{23}^{-1}, Y_{3}\right) .
\end{gathered}
$$


To write the versions $\mathcal{C}_{\|}, \mathcal{C}_{\bullet}, \mathcal{C}_{+}$of the matrix factorizations from above we need more precise notations for the Koszul matrix factorizations. We use the matrix notation

$$
\left[\begin{array}{ccc}
a_{1} & b_{1} & \theta_{1} \\
\vdots & \vdots & \vdots \\
a_{m} & b_{m} & \theta_{m}
\end{array}\right] .
$$

for the matrix factorization from $\operatorname{MF}(X, F)$ with the differential $D=\sum_{i=1}^{m} a_{i} \theta_{i}+b_{i} \frac{\partial}{\partial \theta_{i}}$ acting on $\mathbb{C}[X] \otimes \Lambda^{\bullet}[\theta]$.

Let us also fix coordinates on the space $\mathcal{X}^{\circ}=\mathfrak{g} \times \mathfrak{b} \times G \times \mathfrak{b}$ :

$$
X=\left[\begin{array}{cc}
x_{0}+\operatorname{tr} / 2 & x_{1} \\
x_{-1} & -x_{0}+\operatorname{tr} / 2
\end{array}\right], \quad Y_{i}=\left[\begin{array}{cc}
0 & y_{i} \\
0 & 0
\end{array}\right] \quad g=\left[\begin{array}{cc}
a_{11} & a_{12} \\
a_{21} & a_{22}
\end{array}\right],
$$

where $\operatorname{tr}=\operatorname{tr} X$. We also denote by $\delta_{1}, \delta_{2}$ the generators of Lie $\left(U^{2}\right), U^{2} \subset B^{2}$. We also only indicate non-trivial actions of $\delta_{i}$, that is if no action of $\delta_{i}$ is given then this action is trivial.

With this conventions we have the matrix factorization of the identity braid has the form

$$
\mathcal{C}_{\|}=\left[\begin{array}{ccc}
x_{-1} & y_{1}-y_{2} a_{11}^{2} & \theta_{1} \\
y_{2} \tilde{x}_{0} & a_{21} & \theta_{2}
\end{array}\right], \quad \delta_{1} \theta_{1}=-2 y_{2} a_{11} \theta_{2}
$$

The blob matrix factorization has the form

$$
\mathcal{C}_{\bullet}=\left[\begin{array}{ccc}
x_{-1} & y_{1}-y_{2} a_{11}^{2} & \theta_{1} \\
a_{21} \tilde{x}_{0} & y_{2} & \theta_{2}^{\prime}
\end{array}\right], \quad \delta_{1} \theta_{1}=-2 a_{21} a_{11} \theta_{2}^{\prime}
$$

or equivalently

$$
\mathcal{C} \bullet=\left[\begin{array}{ccc}
x_{-1} & y_{1} & \theta_{1}^{\prime} \\
-a_{11}^{2} x_{-1}+a_{21} \tilde{x}_{0} & y_{2} & \theta_{2}^{\prime}
\end{array}\right], \quad \theta_{1}^{\prime}=\theta_{1}+a_{22}^{2} \theta_{2}^{\prime}, \quad \delta_{1} \theta_{1}^{\prime}=0
$$

The matrix factorization of the positive intersection is

$$
\mathcal{C}_{+}=\left[\begin{array}{ccc}
x_{-1} & y_{1}-y_{2} a_{11}^{2} & \theta_{1} \\
\tilde{x}_{0} & a_{21} y_{2} & \theta_{2}
\end{array}\right], \quad \delta_{1} \theta_{1}=-2 a_{11} \theta_{2}
$$

6.3. Computation. Now we are ready to do our sample computation.

Proposition 6.3.1. In the convolution algebra of $\mathrm{MF}_{\mathrm{GL}_{n} \times B^{2}}(\mathcal{X}, W)$ we have:

$$
\mathcal{C}_{\bullet}\left\langle 0, \chi_{1}\right\rangle \star \mathcal{C}_{\bullet}\left\langle 0, \chi_{1}\right\rangle=\mathcal{C}_{\bullet}\left\langle\chi_{1}, \chi_{1}\right\rangle \oplus \mathcal{C} \bullet\left\langle\chi_{2}, \chi_{1}\right\rangle .
$$

Proof. Let us fix some more notation for the coordinates on the spaces that appear in our constructions. For the group elements elements in the product $\mathcal{X}_{\text {conv }}^{\circ}=\mathfrak{g} \times \mathfrak{n} \times \mathrm{GL}_{2} \times \mathfrak{n} \times \mathrm{GL}_{2} \times \mathfrak{n}$ we use notations $a, b$ and for the non-zero elements of upper-triangular matrices in the product we use $y_{1}, y_{2}, y_{3}$. We also add prime to the conjugate of $X: X^{\prime}=\operatorname{Ad}_{a} X$.

Thus the matrix factorization $\mathcal{C}^{\prime \prime}=\pi_{12}^{\circ *}\left(\mathcal{C}_{\bullet}\right) \otimes \pi_{23}^{\circ *}\left(\mathcal{C}_{\bullet}\right)$ is the following Koszul matrix factorization:

$$
\mathcal{C}^{\prime \prime}=\left[\begin{array}{ccc}
x_{-1} & y_{1}-y_{2} a_{11}^{2} & \theta_{1} \\
a_{21}\left(2 x_{0} a_{11}+x_{1} a_{21}\right) & y_{2} & \theta_{2} \\
x_{-1}^{\prime} & y_{2}-y_{3} b_{11}^{2} & \theta_{3} \\
b_{21}\left(2 x_{0}^{\prime} b_{11}+x_{1}^{\prime} b_{21}\right) & y_{3} & \theta_{4}
\end{array}\right], \quad \delta_{1} \theta_{1}=-2 a_{11} \theta_{2}, \quad \delta_{2} \theta_{3}=-2 b_{11} \theta_{4} .
$$


By making suitable linear change of $\theta_{1} \mapsto \theta_{1}+2 a_{11} \theta_{2}, \theta_{2} \mapsto \theta_{2}$ and $\theta_{3} \mapsto \theta_{3}+b_{11} \theta_{4}, \theta_{4} \mapsto \theta_{4}$ we can make the first simplification of this matrix factorization:

$$
\mathcal{C}^{\prime \prime}=\left[\begin{array}{ccc}
x_{-1} & y_{1} & \theta_{1} \\
-a_{11}^{2} x_{-1}+a_{21}\left(2 x_{0} a_{11}+x_{1} a_{21}\right) & y_{2} & \theta_{2} \\
x_{-1}^{\prime} & y_{2} & \theta_{3} \\
-b_{11}^{2} x_{-1}^{\prime}+b_{21}\left(2 x_{0}^{\prime} b_{11}+x_{1}^{\prime} b_{21}\right) & y_{3} & \theta_{4}
\end{array}\right], \quad \delta_{i} \theta_{j}=0
$$

We use the third row to remove $y_{2}$ from the other rows:

$$
\mathcal{C}^{\prime \prime}=\left[\begin{array}{ccc}
x_{-1} & y_{1} & \theta_{1} \\
-a_{11}^{2} x_{-1}+a_{21}\left(2 x_{0} a_{11}+x_{1} a_{21}\right) & 0 & \theta_{2}^{\prime} \\
0 & y_{2} & \theta_{3} \\
-b_{11}^{2} x_{-1}^{\prime}+b_{21}\left(2 x_{0}^{\prime} b_{11}+x_{1}^{\prime} b_{21}\right) & y_{3} & \theta_{4}
\end{array}\right], \quad \theta_{2}^{\prime}=\theta_{2}-\theta_{3} .
$$

Since $\theta_{3}$ is $B^{2}$ invariant element, we can now remove the third row altogether and work over the ring $R^{\prime}=\mathbb{C}\left[\mathcal{X}_{\text {conv }}^{\circ}\right] /\left(y_{2}\right)$.

We can also use the relation

$$
-b_{11}^{2} x_{-1}^{\prime}+b_{21}\left(2 x_{0}^{\prime} b_{11}+x_{1}^{\prime} b_{21}\right)=-c_{11}^{2} x_{-1}+c_{21}\left(2 x_{0} c_{11}+x_{1} c_{21}\right)
$$

to arrive to

$$
\mathcal{C} \bullet \star \mathcal{C} \bullet=\left[\begin{array}{ccc}
x_{-1} & y_{1} & \theta_{1} \\
-a_{11}^{2} x_{-1}+a_{21}\left(2 x_{0} a_{11}+x_{1} a_{21}\right) & 0 & \theta_{2}^{\prime} \\
-c_{11}^{2} x_{-1}+c_{21}\left(2 x_{0} c_{11}+x_{1} c_{21}\right) & y_{3} & \theta_{4}
\end{array}\right]
$$

Doing couple more simple row transformations, that change the basis in the space $\left\langle\theta_{1}, \theta_{2}^{\prime}, \theta_{4}^{\prime}\right\rangle$, we arrive to a simplified presentation of $\mathcal{C}_{\bullet} \star \mathcal{C}_{\bullet}$ :

$$
\mathcal{C}^{\prime \prime}=\left[\begin{array}{ccc}
x_{-1} & y_{1}-c_{11}^{2} y_{3} & \theta_{1}^{\prime} \\
a_{21}\left(2 x_{0} a_{11}+x_{1} a_{21}\right) & 0 & \theta_{2}^{\prime \prime} \\
c_{21}\left(2 x_{0} c_{11}+x_{1} c_{21}\right) & y_{3} & \theta_{4}^{\prime}
\end{array}\right]
$$

Now let us notice that the top and the bottom lines of the last Koszul complex are $\delta_{2}$ invariant and together they form a Koszul matrix factorization $\pi_{13}^{\circ, *}\left(\mathcal{C}_{\bullet}\right)$. On the other hand the middle line has only one non-trivial differential and to complete the proof we need to compute the Chevalley-Eilenberg homology

$$
H_{\text {Lie }}^{*}\left(\mathfrak{n}, R^{\prime \prime} \stackrel{f}{\rightarrow} R^{\prime \prime}\right)^{T}, \quad f=-a_{21}\left(2 x_{0} a_{11}+x_{1} a_{21}\right),
$$

where $R^{\prime}=R^{\prime \prime} \otimes \mathbb{C}\left[\mathrm{GL}_{2}\right]$ with last copy of $\mathrm{GL}_{2}$ has coordinates $c_{i j}$.

The space $\operatorname{Spec}\left(R^{\prime \prime}\right)$ has coordinates $a, y_{1}, y_{2}, x$ and the Lie algebra $\mathfrak{n}$ only acts on the entries of the matrix $a$ :

$$
\delta_{2} a_{i 2}=-a_{i 1}, \quad \delta_{2} a_{i 1}=0 .
$$

The differential in complex for $H_{\mathrm{Lie}}^{*}$ is exactly $\delta_{2}$ hence

$$
H^{0}\left(\mathfrak{n}, R^{\prime \prime}\right)=\mathbb{C}\left[y_{1}, y_{2}, x, a_{11}, a_{21}, \operatorname{det}^{ \pm 1}\right], \quad H^{1}\left(\mathfrak{n}, R^{\prime \prime}\right)=\mathbb{C}\left[y_{1}, y_{2}, x, a, \operatorname{det}^{ \pm 1}\right] /\left(a_{11}, a_{21}\right),
$$

where $\operatorname{det}=\operatorname{det} a$. Now we can extract the torus invariant part:

$$
\left(H^{*}\left(\mathfrak{n}, R^{\prime \prime}\right) \otimes \chi_{1}\right)^{T_{s c}}=\left(H^{0}\left(\mathfrak{n}, R^{\prime \prime}\right) \otimes \chi_{1}\right)^{T_{s c}}=\left\langle a_{11}, a_{21}\right\rangle .
$$

Finally, let us observe that the function $f$ is quadratic on $a$ hence its induced action action on $\left(H^{*}\left(\mathfrak{n}, R^{\prime \prime}\right) \otimes \chi_{1}\right)^{T_{s c}}$ is trivial and the statement follows. 
Now let us derive the formula (1.2) from the above computation. For that let us recall the stable locus $\overline{\mathcal{X}}^{s t}$ is union of two open subsets: $U_{y}=\{y \neq 0\}, U_{x}=\left\{\left(\operatorname{Ad}_{g}^{-1} X\right)_{12} \neq 0\right\}$. On the open set $U_{y}$ the matrix factorization $\overline{\mathcal{C}}_{\bullet}$ contracts since $y$ is one of the differentials of the curved complexes. Thus we can safely restrict our attention to the open locus $U_{x}$ but on this locus $\left(\operatorname{Ad}_{g}^{-1} X\right)_{12} \neq 0$. Since the weights of $T_{s c} \times B^{2}$ on this non-vanishing elements are:

$$
\text { weight }\left(\left(\operatorname{Ad}_{g}^{-1} X\right)_{12}\right)=\mathbf{q}^{2} \cdot\left\langle 0,-\chi_{1}+\chi_{2}\right\rangle, \quad \text { weight }(\operatorname{det}(a))=\left\langle\chi_{1}+\chi_{2}, \chi_{1}+\chi_{2}\right\rangle
$$

we can trade the Borel action weight for $\mathbf{q} \chi_{1}$-shifts for $\mathbf{q}$-shifts:

$$
\begin{gathered}
\overline{\mathcal{C}} \cdot\left\langle\chi^{\prime}+\chi_{1}, \chi^{\prime \prime}\right\rangle=\mathbf{q}^{2} \overline{\mathcal{C}} \bullet\left\langle\chi^{\prime}-\chi_{2}, \chi^{\prime \prime}-2 \chi_{2}\right\rangle, \\
\overline{\mathcal{C}} \cdot\left\langle\chi^{\prime}, \chi^{\prime}+\chi_{1}\right\rangle=\mathbf{q}^{-2} \overline{\mathcal{C}}_{\bullet}\left\langle\chi^{\prime}, \chi^{\prime \prime}+\chi_{2}\right\rangle .
\end{gathered}
$$

Finally, we refer to the theorem 8.6.1 that implies that the pull-back $j_{s t}^{*}$ turns the shifts $\left\langle\chi_{2}, 0\right\rangle$ and $\left\langle 0, \chi_{2}\right\rangle$ to the trivial $B^{2}$-equivariant shift.

\section{MARKOV RELATIONS}

The first Markov relation is equivalent to $\mathrm{HHH}$ being a trace, that is we need to show that the functor $\mathrm{HHH}$ is constant on the conjugacy classes inside $\mathfrak{B r}_{n}$. In fact one can show stronger statement. Before we state the this stronger statement let us discuss the connection with usual flag Hilbert schemes.

7.1. Sheaves on the flag Hilbert scheme. The usual flag Hilbert scheme FHilb $_{n}$ is a subvariety of Hilb $\mathrm{f}_{1, n}^{\text {ree }}$ defined by the commutativity constraint on $X, Y$ :

$$
[X, Y]=0 .
$$

It turns out that the support of the homology of the complex $\mathbb{S}_{\beta}$ is contained in $\operatorname{Hilb}_{1, n}^{L}$. Hence the sheaf homology of the complex is the sheaf

$$
\mathcal{S}_{\beta}=\mathcal{S}_{\beta}^{\text {odd }} \oplus \mathcal{S}_{\beta}^{\text {even }}:=\mathcal{H}^{*}\left(\text { FHilb }{ }_{n}^{\text {free }}, \mathbb{S}_{\beta}\right)
$$

on Hilb ${ }_{1, n}$ and we immediately have the following:

Theorem 7.1.1. There is a spectral sequence with $E_{2}$ term being

$$
\begin{gathered}
\left(\mathrm{H}^{*}\left(\mathrm{FHilb}_{n}, \mathcal{S}_{\beta} \otimes \Lambda^{k} \mathcal{B}\right), d\right) \\
d: \mathrm{H}^{k}\left(\mathrm{FHilb}_{n}, \mathcal{S}_{\beta}^{\text {odd } / \text { even }} \otimes \Lambda^{k} \mathcal{B}\right) \rightarrow \mathrm{H}^{k-1}\left(\text { FHilb }_{1, n}, \mathcal{S}_{\beta}^{\text {even } / \text { odd }} \otimes \Lambda^{k} \mathcal{B}\right),
\end{gathered}
$$

that converges to $\mathbb{H}^{k}(\beta)$.

The theorem follows almost immediately from the main theorem 4.1 .2 and the proposition 2.1.4. Moreover the sheaf $\mathcal{S}_{\beta}$ is actually is a conjugacy invariant:

Theorem 7.1.2. OR18e For any $\alpha, \beta \in \mathfrak{B r}_{n}$ we have:

$$
\mathcal{S}_{\alpha \cdot \beta} \simeq \mathcal{S}_{\beta \cdot \alpha} .
$$

The argument could be found in the cited paper, here we illustrate the idea by showing that

$$
\mathcal{S}_{\sigma_{i} \sigma_{j} \sigma_{k}} \simeq \mathcal{S}_{\sigma_{j} \sigma_{k} \sigma_{i}}
$$


Indeed, let us introduce the space $\mathcal{X}_{3} \subset \mathfrak{g l}_{n} \times\left(\mathrm{GL}_{n} \times \mathfrak{n}_{n}\right)^{3}$ defined by the constraint requiring the cyclic product of the group elements to be one. There is a natural $B^{3}$-action and $B^{3}$ equivariant projections :

$$
p r_{i}: \mathcal{X}_{3} \rightarrow \mathfrak{g l}_{n} \times \mathfrak{n}_{n}, \quad \operatorname{pr}_{i}\left(X, g_{1}, Y_{1}, g_{2}, Y_{2}, g_{3}, Y_{3}\right)=\left(X, Y_{i}\right) .
$$

Respectively, we also have projections $\pi_{12}^{\circ}, \pi_{23}^{\circ}, \pi_{31}^{\circ}: \mathcal{X}_{3} \rightarrow \mathcal{X}^{\circ}$ and

$$
\mathbb{S}_{\sigma_{i} \sigma_{j} \sigma_{k}}=\operatorname{pr}_{1 *}(\mathcal{C}), \quad \mathbb{S}_{\sigma_{j} \sigma_{k} \sigma_{i}}=\operatorname{pr}_{2 *}(\mathcal{C}), \quad \mathcal{C}=\operatorname{CE}_{\mathfrak{n}^{3}}\left(\pi_{12}^{\circ *}\left(\mathcal{C}_{+}^{(i)}\right) \otimes \pi_{23}^{\circ *}\left(\mathcal{C}_{+}^{(j)}\right) \otimes \pi_{31}^{\circ *}\left(\mathcal{C}_{+}^{(k)}\right)\right)
$$

On the critical locus of $\pi_{i, i+1}^{\circ *}\left(W^{\circ}\right)$ we have $Y_{i}=\operatorname{Ad}_{g_{i}} Y_{i+1}$ hence on the critical locus the conjugation by $g_{1}$ intertwines the projections $p r_{1}$ and $p r_{2}$ the isomorphism (7.1) follows.

In the argument above we ignore the stability conditions but can check that the shrinking lemma 3.4 .2 implies that the argument above works even after we impose the stability conditions.

7.2. Second Markov move. The second Markov relation is more subtle and a proof of this relation is arguably the most valuable result of [OR18e]. To convey the main idea of the proof we explain why it holds for the braids on two strands. In this case we need to compare the homology of the closure of $\sigma_{1}^{ \pm 1}$ with the homology of unknot, so let us first do the most trivial case of the braids on one strands since $\mathrm{L}\left(\mathbf{1}_{1}\right), \mathbf{1}_{1} \in \mathfrak{B r}_{1}$ is manifestly the unknot.

Indeed, for $n=1$ we have $\overline{\mathcal{X}}_{1}=\mathbb{C} \times \mathbb{C}^{*} \times 0$ and $j_{e}$ embeds $\widetilde{\text { FHilb }}_{1}^{\text {free }}=\mathbb{C} \times 1 \times 0$ inside $\overline{\mathcal{X}}_{1}$. The group $B_{1}=\mathbb{C}^{*}$ acts trivially on $\widetilde{\text { FHilb }}_{1}^{\text {free }}$ and thus FHilb $1=\mathbb{C}$ and $\mathbb{S}_{e}=j_{e}^{*}\left(\mathcal{O}_{\overline{\mathcal{X}}_{1}}\right)=\mathcal{O}_{\mathbb{C}}$ and $\mathcal{B}_{1}$ is the trivial bundle. We conclude then:

$$
\operatorname{dim}_{q, t} H^{0}\left(\mathbf{1}_{1}\right)=\operatorname{dim}_{q, t} H^{1}\left(\mathbf{1}_{1}\right)=\frac{1}{1-q^{2}} .
$$

Now let us explore the geometry of the free Hilbert scheme FHilb ${ }_{2}^{\text {free }}$. Let us fix coordinates on the space $\widetilde{\mathrm{FHilb}}_{2}^{\text {free }} \subset \mathfrak{b} \times \mathfrak{n} \times V$ :

$$
X=\left[\begin{array}{cc}
x_{11} & x_{12} \\
0 & x_{22}
\end{array}\right], \quad Y=\left[\begin{array}{cc}
0 & y \\
0 & 0,
\end{array}\right], \quad v=\left[\begin{array}{l}
v_{1} \\
v_{2}
\end{array}\right] .
$$

Since we have the stability condition $\mathbb{C}\langle X, Y\rangle v=\mathbb{C}^{2}$ and both $X, Y$ are upper-triangular, we must have $v_{2} \neq 0$. Thus after conjugating by the appropriate upper-triangular matrix we could assume that $v_{2}=1, v_{1}=0$, let us denote this vector by $v^{0}$. It is also elementary to see that

$$
\mathbb{C}\langle X, Y\rangle v^{0}=\mathbb{C}^{2} \text { if and only if } x_{12} y \neq 0 .
$$

Also the stabilizer of $v^{0}$ is $\mathbb{C}^{*}$ that scales $x_{12}, y$ and preserves $x_{11}, x_{22}$. Hence we have shown:

$$
\mathrm{FHilb}_{2}^{\text {free }}=\mathbb{P}^{1} \times \mathbb{C}^{2},
$$

the projection $p$ on $\mathbb{C}^{2}$ is given by the coordinates $x_{11}, x_{22}$.

Let us contrast the geometry of FHilb 2 free with the geometry of FHilb 2 . The discussion in this paragraph is not used in the proof below and is just an illustration of difficulties of the geometry of the flag Hilbert scheme. The condition $[X, Y]=0$ is equivalent to the constraint:

$$
y\left(x_{11}-x_{22}\right)=0 .
$$

Hence the fibers of the projection $p:$ FHilb $_{2} \rightarrow \mathbb{C}^{2}$ are points outside of the diagonal $x_{11}=x_{22}$ and the fibers are projective lines $\mathbb{P}^{1}$ over the diagonal. 
Next let us recall that the matrix factorization for the simple positive crossing is $\mathcal{C}_{+}=$ $\left|\tilde{x}, y g_{21}\right|$. Since $\left.\tilde{x}\right|_{g=1}=\left(x_{11}-x_{22}\right)$, the pull-back $j_{e}\left(\mathcal{C}_{+}\right)$is the Koszul complex that is homotopy equivalent to the structure sheaf of $\mathbb{P}^{1} \times \mathbb{C}$. Finally, the tautological vector bundle is a sum of the line bundles $\mathcal{B}^{\vee}=\mathcal{O} \oplus \mathcal{O}(-1)$, hence:

$$
\begin{gathered}
H^{0}\left(\sigma_{1}\right)=H^{*}\left(\mathcal{O}_{\mathbb{P}^{1} \times \mathbb{C}}\right)=\mathbb{C}\left[x_{11}\right], \quad H^{1}\left(\sigma_{1}\right)=H^{*}\left(\mathcal{B}^{\vee}\right)=H^{*}\left(\mathcal{O}_{\mathbb{P}^{1} \times \mathbb{C}} \oplus \mathcal{O}_{\mathbb{P}^{1} \times \mathbb{C}}(-1)\right)=\mathbb{C}\left[x_{11}\right], \\
H^{2}(\sigma)=H^{*}(\operatorname{det}(\mathcal{B}))=H^{*}\left(\mathcal{O}_{\mathbb{P}^{1} \times \mathbb{C}}(-1)\right)=0 .
\end{gathered}
$$

By our construction the matrix factorization for the negative crossing is differs by a line bundle twist from the one for positive crossing. In particular, we have $j_{e}\left(\mathcal{C}_{-}\right)=\mathcal{O}_{\mathbb{P}^{1} \times \mathbb{C}}(-1)$ and can compute the homology:

$$
\begin{gathered}
H^{0}\left(\sigma_{1}^{-1}\right)=H^{*}\left(\mathcal{O}_{\mathbb{P}^{1} \times \mathbb{C}}(-1)\right)=0, \quad H^{2}\left(\sigma^{-1}\right)=H^{*}(\operatorname{det}(\mathcal{B}) \otimes \mathcal{O}(-1))=H^{*}\left(\mathcal{O}_{\mathbb{P}^{1} \times \mathbb{C}}(-2)\right)=\mathbb{C}\left[x_{11}\right], \\
H^{1}\left(\sigma_{1}^{-1}\right)=H^{*}\left(\mathcal{B}^{\vee} \otimes \mathcal{O}(-1)\right)=H^{*}\left(\mathcal{O}_{\mathbb{P}^{1} \times \mathbb{C}}(-1) \oplus \mathcal{O}_{\mathbb{P}^{1} \times \mathbb{C}}(-2)\right)=\mathbb{C}\left[x_{11}\right],
\end{gathered}
$$

Thus we have shown $H^{k}(\sigma)=H^{k}\left(\mathbf{1}_{1}\right)$ and $H^{k+1}\left(\sigma^{-1}\right)=H^{k}\left(\mathbf{1}_{1}\right)$ as we expected.

Respectively, we can use nested nature of the scheme $\mathrm{FHilb}_{n}$ to define the intermediate map:

$$
\pi: \text { FHilb }_{n}^{\text {free }} \rightarrow \mathbb{C} \times \text { FHilb }_{n-1}^{\text {free }},
$$

where the first component of the map $\pi$ is $x_{11}$ and the second component is just forgetting of the first rows and rows of the matrices $X, Y$ and the first component of the vector $v$. Let us also fix notation for the line bundles on $\mathrm{FHilb}_{n}^{\text {free }}$ : we denote by $\mathcal{O}_{k}(-1)$ the line bundle induced from the twisted trivial bundle $\mathcal{O} \otimes \chi_{k}$. It is quite elementary to show

Proposition 7.2.1. The fibers of the map $\pi$ are projective spaces $\mathbb{P}^{n-1}$ and

(1) $\mathcal{B}_{n} / \pi^{*}\left(\mathcal{B}_{n-1}\right)=\mathcal{O}_{n}(-1)$.

(2) $\left.\mathcal{O}_{n}(-1)\right|_{\pi^{-1}(z)}=\mathcal{O}_{\mathbb{P}^{n-1}}(-1)$.

We can combine the last proposition with the observation that the total homology $H^{*}\left(\mathbb{P}^{n-1}, \mathcal{O}(-l)\right)$ vanish if $l \in(1, n-1)$ and is one-dimensional for $l=0, n$ :

Corollary 7.2.2. For any $n$ we have:

- $\pi_{*}\left(\Lambda^{k} \mathcal{B}_{n}\right)=\Lambda^{k} \mathcal{B}_{n-1}$

- $\pi_{*}\left(\mathcal{O}_{n}(m) \otimes \Lambda^{k} \mathcal{B}_{n}\right)=0$ if $m \in[-n+2,-1]$.

- $\pi_{*}\left(\mathcal{O}_{n}(-n+1) \otimes \Lambda^{k} \mathcal{B}_{n}\right)=\Lambda^{k-1} \mathcal{B}_{n-1}[n]$

The geometric version of the Markov move is the following

Theorem 7.2.3. For any $\beta \in \mathfrak{B r}_{n-1}$ we have

$$
\mathbb{H}^{k}\left(\beta \cdot \sigma_{1}\right)=\mathbb{H}^{k}(\beta), \quad \mathbb{H}^{k}\left(\beta \cdot \sigma_{1}\right)=\mathbb{H}^{k-1}(\beta) .
$$

Sketch of a proof. The main technical component of the proof is the careful analysis of the matrix factorizations $\overline{\mathcal{C}}_{\beta \cdot \sigma^{ \pm 1}} \operatorname{MF}\left(\overline{\mathcal{X}}_{n}, \bar{W}\right)$. It is shown in OR18e that this curved complex $\overline{\mathcal{C}}_{\beta \cdot \sigma_{1}^{\epsilon}}$ has form:

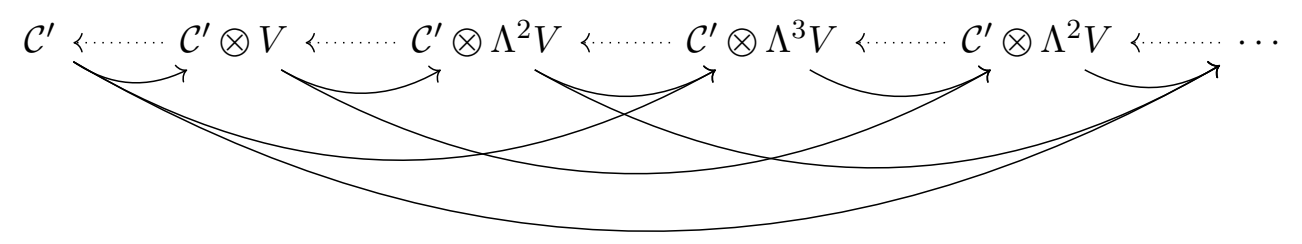


where $\mathcal{C}^{\prime}=p_{1}^{*}\left(\overline{\mathcal{C}}_{\beta}\right), V=\mathbb{C}^{n-2}$, the dotted arrows are the differentials of the Koszul complex for the ideal $I=\left(g_{13}, \ldots, g_{1 n}\right)$ where $g_{i j}$ are the coordinates on the group inside the product $\overline{\mathcal{X}}_{n}=\mathfrak{b}_{n} \times \mathrm{GL}_{n} \times \mathfrak{n}_{n}$. Thus after the pull-back $j_{e}^{*}$ the dotted arrows of the curved complex vanish and we only left with the arrows going from the left to right.

Now we would like to compute $\pi_{*}\left(j_{e}^{*}\left(\overline{\mathcal{C}}_{\beta \cdot \sigma_{1}^{ \pm 1}}\right) \otimes \Lambda^{k} \mathcal{B}_{n}\right)$ and here we can apply the previous corollary. Thus if $\epsilon=1$ then only the left extreme term of $j_{e}^{*}$ of the complex (7.2) survive the push-forward $\pi_{*}$. Since the non-trivial arrows of $j_{e}^{*}$ of (7.2) all are the solid arrows which are going the left to the right, the contraction of the $\pi_{*}$-acyclic terms do not lead to appearance of new correction arrows thus conclude that

$$
\pi_{*}\left(j_{e}^{*}\left(\overline{\mathcal{C}}_{\beta \cdot \sigma_{1}}\right) \otimes \Lambda^{k} \mathcal{B}_{n}\right)=j_{e}^{*}\left(\overline{\mathcal{C}}_{\beta} \otimes \Lambda^{k} \mathcal{B}_{n-1}\right) .
$$

If $\epsilon=-1$ then only the right extreme term of $j_{e}^{*}$ of the complex (17.2) survive the pushforward $\pi_{*}$. Hence the similar argument as before implies:

$$
\pi_{*}\left(j_{e}^{*}\left(\overline{\mathcal{C}}_{\beta \cdot \sigma_{1}^{-1}}\right) \otimes \Lambda^{k} \mathcal{B}_{n}\right)=j_{e}^{*}\left(\overline{\mathcal{C}}_{\beta} \otimes \Lambda^{k-1} \mathcal{B}_{n-1}\right)
$$

\section{ChERN FUnCtOR AND LOCALiZATION}

The theorem 7.1.1 provides a theoretical method for constructing a sheaf on the flag Hilbert scheme that contains all the information about the knot homology of the closure of the braid $\mathrm{L}(\beta)$. However, it is hard to use this method for actually computing knot homology.

The first complication comes from the fact that the space FHilb ${ }_{n}$ is very singular and working with this space requires extra level of care and technicalities [GRN16]. We will explain how one can circumvent this complication with the Chern functor from the next subsection.

The second complication comes from possible non-vanishing of the differential $d$ in the theorem, one would like to avoid the spectral sequence that do not degenerate at the second step. The differential vanishes automatically if for example $\mathcal{S}_{\beta}^{\text {odd }}$ vanishes, this kind of property is probably related to the parity property in [EH17a] for Soergel bimodel model of the knot homology. Again the Chern functor helps with finding braids that have the parity property, as we explain in the end of the section.

8.1. Chern functor. In the paper [OR18d] we construct a pair of functors which we call a Chern functor and a co-Chern functor:

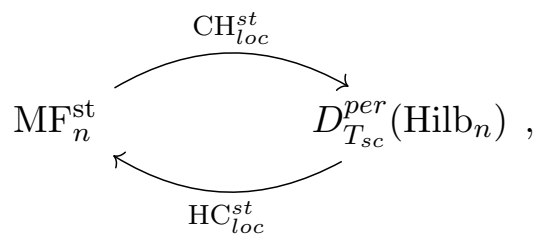

where $\mathrm{Hilb}_{n}$ is the Hilbert scheme of $n$ points on $\mathbb{C}^{2}$, while $D_{T_{s c}}^{\text {per }}\left(\mathrm{Hilb}_{n}\right)$ is the derived category of two-periodic $T_{s c}$-equivariant complexes on the Hilbert scheme. In the same paper we prove

Theorem 8.1.1. OR18d] For every $n$ we have

- The functors $\mathrm{CH}_{\mathrm{loc}}^{\mathrm{st}}$ and $\mathrm{HC}_{\mathrm{loc}}^{\mathrm{st}}$ are adjoint.

- The functor $\mathrm{HC}_{\mathrm{loc}}^{\mathrm{st}}$ is monoidal.

- The image of $\mathrm{HC}_{\mathrm{loc}}^{\mathrm{st}}$ commutes with the elements $\Phi(\beta), \beta \in \mathfrak{B r}_{n}$. 
As a manifestation of the categorified Riemann-Roch formula, we obtain a new interpretation for the triply-graded homology:

Theorem 8.1.2. OR18d For any $\beta \in \mathfrak{B r}_{n}$ we have:

$$
\operatorname{HHH}(\beta)=\operatorname{Hom}\left(\mathcal{O}, \mathrm{CH}_{\text {loc }}^{\text {st }}(\Phi(\beta)) \otimes \Lambda^{\bullet} \mathcal{B}\right) .
$$

Let us outline the construction of the Chern functor in the next subsection.

8.2. The Chern CH. First we will construct the functor between the categories MF and $\mathrm{MF}_{\mathrm{Dr}}$ where the last category is defined as a stable version of the category of equivariant matrix factorizations:

$$
\mathrm{MF}_{\mathrm{Dr}}:=\operatorname{MF}_{G}\left(\mathscr{C}, W_{\text {Dr }}\right), \quad \mathscr{C}=\mathfrak{g} \times G \times \mathfrak{g}, \quad W_{\mathrm{Dr}}(Z, g, X)=\operatorname{Tr}\left(X\left(Z-\operatorname{Ad}_{g} Z\right)\right),
$$

the group $\mathrm{GL}_{n}$ acting on components of $\mathscr{C}$ by conjugation. The stable version of the category is defined as category of matrix factorizations on the slightly enlarged space:

$$
\mathscr{C}^{s t} \subset \mathscr{C} \times V, \quad(Z, g, X, v) \in \mathscr{C}^{s t} \text { iff } g v=v .
$$

Both stable and unstable versions of the categories fit into the diagram:

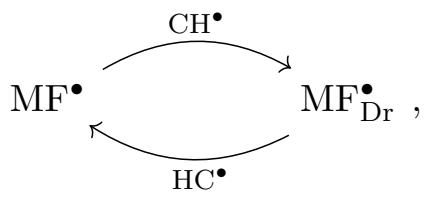

where $\bullet$ can be either st or $\varnothing$.

To lighten the exposition we explain only the construction for the functors $\mathrm{CH}$ and $\mathrm{HC}$, the stable version is an easy modification of the construction, see OR18d]. We need two auxiliary spaces in order to define the Chern functor:

$$
\mathscr{Z}_{\mathrm{CH}}^{0}=\mathfrak{g} \times G \times \mathfrak{g} \times G \times \mathfrak{n}, \quad \mathscr{Z}_{\mathrm{CH}}=\mathfrak{g} \times G \times \mathfrak{g} \times G \times \mathfrak{b}
$$

The action of $G \times B$ on these spaces is

$$
(k, b) \cdot(Z, g, X, h, Y)=\left(\operatorname{Ad}_{k}(Z), \operatorname{Ad}_{k}(g), \operatorname{Ad}_{k}(X), k h b, \operatorname{Ad}_{b^{-1}}(Y)\right)
$$

and the invariant potential is

$$
W_{\mathrm{CH}}(Z, g, X, h, Y)=\operatorname{Tr}\left(X\left(\operatorname{Ad}_{g h}(Y)-\operatorname{Ad}_{h}(Y)\right)\right) .
$$

The spaces $\mathscr{C}$ and $\mathscr{X}$ are endowed with the standard $G \times B^{2}$-equivariant structure, the action of $B^{2}$ on $\mathscr{C}$ is trivial. The following maps

$$
\begin{gathered}
\pi_{\mathrm{Dr}}: \mathscr{Z}_{\mathrm{CH}} \rightarrow \mathscr{C}, \quad f_{\Delta}: \mathscr{Z}_{\mathrm{CH}}^{0} \rightarrow \mathscr{X} \quad j^{0}: \mathscr{Z}_{\mathrm{CH}}^{0} \rightarrow \mathscr{Z}_{\mathrm{CH}} . \\
\pi_{\mathrm{Dr}}(Z, g, X, h, Y)=(Z, g, X), \quad f_{\Delta}(Z, g, X, h, Y)=(X, g h, Y, h, Y)
\end{gathered}
$$

are fully equivariant if we restrict the $B^{2}$-equivariant structure on $\mathscr{X}$ to the $B$-equivariant structure via the diagonal embedding $\Delta: B \rightarrow B^{2}$. Note that $j^{0}$ is the inclusion map.

The kernel of the Fourier-Mukai transform is the Koszul matrix factorization

$$
\mathrm{K}_{\mathrm{CH}}:=\left[X-\operatorname{Ad}_{g^{-1}} X, \operatorname{Ad}_{h} Y-Z\right] \in \operatorname{MF}\left(\mathscr{Z}_{\mathrm{CH}}, \pi_{\mathrm{Dr}}^{*}\left(W_{\mathrm{Dr}}\right)-f_{\Delta}^{*}(W)\right) .
$$

and we define the Chern functor:

$$
\mathrm{CH}(\mathcal{C}):=\pi_{\text {Dr* }}\left(\mathrm{CE}_{\mathfrak{n}}\left(\mathrm{K}_{\mathrm{CH}} \otimes\left(j_{*}^{0} \circ f_{\Delta}^{*}(\mathcal{C})\right)\right)^{T}\right) .
$$


We also define the co-Chern functor $\mathrm{HC}$ as the adjoint functor that goes in the opposite direction: $\mathrm{HC}: \mathrm{MF}_{\mathrm{Dr}} \rightarrow \mathrm{MF}$. Thus, the functor $\mathrm{HC}$ is the composition of adjoints of all the functors that appear in the formula (8.2).

The product $\mathscr{Z}_{\mathrm{CH}} \times B$ has a $B \times B$-equivariant structure: for $(p, g) \in \mathscr{Z}_{\mathrm{CH}} \times B$ we define

$$
\left(h_{1}, h_{2}\right) \cdot(p, g)=\left(h_{1} \cdot p, h_{1} g h_{2}^{-1}\right)
$$

Then the following map is $B^{2}$-equivariant:

$$
\begin{gathered}
\tilde{f}_{\Delta}: \mathscr{Z}_{\mathrm{CH}}^{0} \times B \rightarrow \mathscr{X} \times B, \\
\tilde{f}_{\Delta}(Z, g, X, h, Y, b)=\left(X, g h, Y, h b, \operatorname{Ad}_{b} Y, b\right) .
\end{gathered}
$$

The map $\tilde{f}_{\Delta}$ is a composition of the projection along the first factor of $\mathscr{Z}_{\mathrm{CH}}$ and the embedding inside $\mathscr{X} \times B$. The embedding is defined by the formula

$$
\operatorname{Ad}_{b} Y_{1}=Y_{2},
$$

so it is a regular embedding. Thus since

$$
j^{0 *}\left(\mathrm{~K}_{\mathrm{CH}} \otimes \tilde{\pi}_{\mathrm{Dr}}^{*}(\mathcal{D})\right) \in \mathrm{MF}_{G \times B^{2}}\left(\mathscr{Z}_{\mathrm{CH}} \times B, \tilde{f}_{\Delta}^{*}(W)\right),
$$

where $\tilde{\pi}_{\mathrm{Dr}}: \mathscr{Z}_{\mathrm{HC}} \times B \rightarrow \mathscr{C}$ is a natural extension of map $\pi_{\mathrm{Dr}}$ by the projection along $B$, we have a well-defined matrix factorization $\tilde{f}_{\Delta *} \circ j^{0 *}\left(\mathrm{~K}_{\mathrm{CH}} \otimes \pi_{\mathrm{Dr}}^{*}(\mathcal{D})\right) \in \mathrm{MF}_{G \times B^{2}}\left(\mathscr{X} \times B, \pi_{B}^{*}(W)\right)$, where $\pi_{B}$ is the projection along the last factor. Now we can define:

$$
\mathrm{HC}(\mathcal{D}):=\pi_{B *}\left(\tilde{f}_{\Delta *} \circ j^{0 *}\left(\mathrm{~K}_{\mathrm{CH}} \otimes \pi_{\mathrm{Dr}}^{*}(\mathcal{D})\right)\right) .
$$

8.3. Linear Koszul duality. We need to relate the category $\mathrm{MF}_{\mathrm{Dr}}^{s t}$ and the category $D_{T_{s c}}^{\text {per }}(\mathrm{Hilb})$. This relation is a particular example of the linear Koszul duality. Let us discuss the linear Koszul duality in general.

Derived algebraic geometry is explained in many places, here we explain it in the most elementary setting sufficient for our needs.

Initial data for an affine derived complete intersection is a collection of elements $f_{1}, \ldots, f_{m} \in$ $\mathbb{C}[X]$. It determines the differential graded algebra

$$
\mathcal{R}=\left(\mathbb{C}[X] \otimes \Lambda^{*} U, D\right), \quad D=\sum_{i=1}^{m} f_{i} \frac{\partial}{\partial \theta_{i}},
$$

where $\theta_{i}$ from a basis of $U=\mathbb{C}^{m}$.

More generally, given a dg algebra $\mathcal{R}$ such that $H^{0}(\mathcal{R})=\mathcal{O}_{Z}$ we say that $\operatorname{Spec}(\mathcal{R})$ is a dg scheme with underlying scheme $Z$. Respectively, we define dg category of coherent sheaves on $\operatorname{Spec}(\mathcal{R})$ as

$$
\operatorname{Coh}(\operatorname{Spec}(\mathcal{R}))=\frac{\{\text { bounded complexes of finitely generated } \mathcal{R} \text { dg modules }\}}{\{\text { quasi-isomorphisms }\}} .
$$

Consider a potential on $X \times U$ :

$$
W=\sum_{i=1}^{m} f_{i}(x) z_{i}
$$

where $z_{i}$ is a basis of $U^{*}$ dual to the basis $\theta_{i}$. For the Koszul matrix factorization:

$$
\operatorname{MF}(X \times U, W) \ni \mathrm{B}=\left(\mathcal{R} \otimes \mathbb{C}[U], D_{B}\right), \quad D_{B}=\sum_{i=1}^{m} z_{i} \theta_{i}+f_{i} \frac{\partial}{\partial \theta_{i}} .
$$


and for a $\left(M, D_{M}\right)$ dg module over $\mathcal{R}$, the tensor product

$$
\mathrm{KSZ}_{U}(M):=M \otimes_{\mathbb{C}[X] \otimes \Lambda^{*}(U)} \mathrm{B}
$$

is an object of $\operatorname{MF}(X \times U, W)$ with the differential $D=D_{M} \otimes 1+1 \otimes D_{B}$. The map $\mathrm{KSZ}_{U}$ extends to a functor between triangulated categories:

$$
\mathrm{KSZ}_{U}: \operatorname{Coh}(\operatorname{Spec}(\mathcal{R})) \rightarrow \operatorname{MF}(X \times U, W) .
$$

The functor in the other direction is based on the dual matrix factorization:

$$
\begin{gathered}
\operatorname{MF}(X \times U,-W) \ni B^{*}=\left(\mathcal{R} \otimes \mathbb{C}[U], D_{B}^{*}\right), \quad D_{B}^{*}=-\sum_{i=1}^{m} z_{i} \theta_{i}+f_{i} \frac{\partial}{\partial \theta_{i}}, \\
\mathrm{KSZ}_{U}^{*}: \operatorname{MF}(X \times U, W) \rightarrow \operatorname{Coh}(\operatorname{Spec}(\mathcal{R})), \quad \operatorname{KSZ}_{U}^{*}(\mathcal{F}):=\operatorname{Hom}_{\mathcal{R}}\left(\mathcal{F} \otimes_{\mathbb{C}[X \times U]} \mathrm{B}^{*}, \mathcal{R}\right) .
\end{gathered}
$$

Theorem 8.3.1. The compositions of the functors:

$$
\mathrm{KSZ}_{U} \circ \mathrm{KSZ}_{U}^{*}, \quad \mathrm{KSZ}_{U}^{*} \circ \mathrm{KSZ}_{U}
$$

are autoequivalences of the corresponding categories.

Proof of this theorem could be found in [Isi13], AK15a] or one can consult [OR18d] for a more streamlined argument.

8.4. Linearization. We would like to apply the Koszul duality in our situation. The complication in our case is that we want to eliminate the group factor in the space $\mathscr{C}^{\text {st }}$ but the group is not a linear space. Thus we have to restrict ourselves to the neighborhood of the identity and linearize the potential in this neighborhood and as we explain below it could be done with localization.

A coordinate substitution $Y=U g^{-1}$ on our main variety $\mathscr{C}$ makes the potential tri-linear:

$$
\underline{W}_{\operatorname{Dr}}(X, U, g)=\operatorname{Tr}(X[U, g])=W_{\operatorname{Dr}}\left(X, U g^{-1}, g\right) .
$$

Thus we introduce linearized categories:

$$
\underline{\operatorname{MF}}_{\mathrm{Dr}}^{\bullet}:=\operatorname{MF}_{G}\left(\underline{\mathscr{C}}^{\bullet}, \underline{W}_{\text {lin }}\right),
$$

where $\mathscr{C}^{\bullet}$ is obtained from $\mathscr{C} \bullet$ by taking the closure of $G$ inside $\mathfrak{g}$.

Since $j_{G}: \mathscr{C} \bullet \underline{\mathscr{C}}^{\bullet}$ is an open embedding, the pull-back functor $j_{G}^{*}$ is a localization functor and we denote

for this functor.

$$
\operatorname{loc}^{\bullet}: \mathrm{MF}_{\mathrm{Dr}}^{\bullet} \rightarrow \underline{\mathrm{MF}_{\mathrm{Dr}}^{\bullet}}
$$

Proposition 8.4.1. OR18d] The functors $\operatorname{loc}^{\text {st }}$ are isomorphisms.

Since the potential $\underline{W}$ is linear as a function of $g \in \mathfrak{g}$ and the scaling torus $T_{s c}$ does not act on $g$, we obtain a pair of mutually inverse functors:

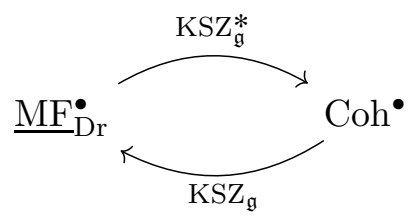

here $\mathrm{Coh}^{s t}$ is the two-periodic derived category $D^{\text {per }}$ (Hilb) and Coh is the DG category of the commuting variety.

The functors that we wanted to construct are defined by the composing the functors: 


$$
\mathrm{CH}_{\mathrm{loc}}^{\mathrm{st}}:=\mathrm{CH}^{\mathrm{st}} \circ\left(\mathrm{loc}^{\mathrm{st}}\right)^{-1} \circ \mathrm{KSZ}_{\mathfrak{g}}^{*}: \mathrm{MF}^{\bullet} \rightarrow \mathrm{D}^{\mathrm{per}}\left(\operatorname{Hilb}_{n}\left(\mathbb{C}^{2}\right)\right) .
$$

The localization functor does not seem to be invertible in the case of $\bullet=\varnothing$, however a construction of the functor in the opposite direction does not require invertibility of the localization:

$$
\mathrm{HC}_{\mathrm{loc}}^{\bullet}:=\mathrm{HC}^{\bullet} \circ \mathrm{loc}^{\bullet} \circ \mathrm{KSZ}_{\mathfrak{g}}: \mathrm{Coh}^{\bullet} \rightarrow \mathrm{MF}^{\bullet}
$$

8.5. Localization formulas. The advantage of this new interpretation is that the Hilbert scheme is smooth, unlike the flag Hilbert scheme which is a homological support of $\mathcal{E} \times(\Phi(\beta), \Phi(1))$. So the complexes on Hilb are more manageable than their flag counter-part. In support of this expectation, we apply the Chern functor to the Jusys-Murphy (JM) subgroup inside $\mathfrak{B r}_{n}$ together with the parity property and prove an explicit localization formula for the sufficiently positive elements of the JM subgroup.

Recall that the JM subgroup is generated by the elements

$$
\delta_{i}=\sigma_{i} \sigma_{i+1} \ldots \sigma_{n-1}^{2} \ldots \sigma_{i+1} \sigma_{i} .
$$

It is not hard to see that these elements mutually commute and that the full twist from the introduction is the product:

$$
\mathrm{FT}=\prod_{i=1}^{n-1} \delta_{i}
$$

It is expected that $\mathrm{CH}_{\text {loc }}^{\text {st }}$ applied to the matrix factorization corresponding to the sufficiently positive element of JM algebra is a sheaf supported in one homological degree, we state the precise conjecture below. Modulo this geometric conjecture we have a (conditional on the conjecture) formula for the corresponding homology of the links.

Theorem* 8.6. For any $n$ there are $N, M>0$ such that for a vector $\vec{b} \in \mathbb{Z}^{n-1}$ with $a_{i+1}-a_{i}>$ $N, a_{2}>M$ the $(q, t, a)$-character of the homology of the closure of the braid $\prod_{i=2} \delta^{b_{i}}$ is given by the formula

$$
\operatorname{dim}_{a, Q, T} \operatorname{HHH}\left(\prod_{i=2}^{n} \delta^{b_{i}}\right)=\sum_{T} \prod_{i} \frac{z_{i}^{b_{i}}\left(1+a z_{i}^{-1}\right)}{1-z^{-1}} \prod_{1 \leqslant i<j \leqslant n} \zeta\left(\frac{z_{i}}{z_{j}}\right),
$$

where $\zeta(x)=\frac{(1-x)(1-Q T x)}{(1-Q x)(1-T x)}, Q=q^{2}, T=t^{2} / q^{2}$. The last sum is over all standard Young tableaux with $z_{i}=Q^{a^{\prime}(i)} T^{l^{\prime}(i)}, a^{\prime}, l^{\prime}$ are co-arm and co-leg of the square the standard tableau with the square with the label $i$.

The proof has two components. The first component is concerned with actual computation of the matrix factorization $\Phi\left(\prod_{i=2} \delta_{i}^{b_{i}}\right)$. This computation is an easy consequence of our construction of $\Phi^{a f f}$ :

Theorem 8.6.1. OR18c For any $i=1, \ldots, n$ we have

$$
\Phi^{a f f}\left(\Delta_{i}\right)=\Phi^{a f f}(1)\left\langle\chi_{i}, 0\right\rangle .
$$

In particular, we show in $\left[\mathrm{OR} 18 \mathrm{c}\right.$ ] that the pull-back $j_{s t}^{*}$ sends $\Phi^{a f f}(1)\left\langle\chi_{i}, 0\right\rangle$ to the trivial line bundle. Since $\delta_{i}=\operatorname{fgt}\left(\Delta_{i}\right)$ we conclude the following 
Corollary 8.6.2. For any $\beta \in \mathfrak{B r}_{n}$ and $b_{i}, M \in \mathbb{Z}$ we have

$$
\begin{gathered}
\Phi\left(\beta \cdot \prod_{i=2}^{n} \delta_{i}^{b_{i}}\right)=\Phi(\beta)\langle\vec{b}, 0\rangle, \\
\mathrm{CH}_{\text {loc }}^{\text {st }}\left(\Phi\left(\beta \cdot \mathrm{FT}^{M}\right)\right)=\mathrm{CH}_{\text {loc }}^{\text {st }}(\Phi(\beta)) \otimes \operatorname{det}(\mathcal{B})^{\otimes M},
\end{gathered}
$$

where $\mathrm{FT}=\prod_{i=2}^{n} \delta_{i}$ is the full-twist braid.

Thus we can apply the first formula from the corollary and get an explicit Koszul matrix factorization describing the desired curved complex:

$$
\Phi\left(\prod_{i=2}^{n} \delta_{i}^{b_{i}}\right)=\mathcal{C}_{\|}\langle\vec{b}, 0\rangle .
$$

It is much harder though to compute Chern functor $\mathrm{CH}\left(\mathcal{C}_{\|}\langle\vec{b}, 0\rangle\right)$. It is expected GRN16 that $\mathrm{CH}\left(\mathcal{C}_{\|}\right)$is a celebrated Procesi vector bundle and finding an explicit description for this vector bundle is notoriously hard [Hai02]. So at the moment we do not have an explicit statement for $\mathrm{CH}\left(\mathcal{C}_{\|}\langle\vec{b}, 0\rangle\right)$ but we believe the following weaker conjecture could be proved by inductive argument from the work of Haiman.

Conjecture 8.6.3. OR18f] There is $N>0$ such that for any $\vec{a}$ such that $a_{i+1}-a_{i}>N$ the two periodic complex $\mathrm{CH}_{\text {loc }}^{\text {st }}\left(\mathcal{C}_{\|}\langle\vec{a}, 0\rangle\right)$ is homotopy equivalent to the sheaf concentrated in even homological degree.

On the other hand $\operatorname{det}(\mathcal{B})$ is an ample line bundle on $\operatorname{Hilb}_{n}\left(\mathbb{C}^{2}\right)$ and hence the assumptions of the theorem 8.6 and corollary 8.6 .2 imply that $\mathrm{CH}_{\text {loc }}^{\text {st }}\left(\mathcal{C}_{\|}\langle\vec{b})\right\rangle$ is homotopy equivalent to the sheaf with no higher homology. The differential in the complex $\mathcal{C}_{\|}$has $T_{s c}$ degree t, respectively by t-twisting even component of $\mathcal{C}_{\|}$we obtain the curved complex $\mathcal{C}_{\|}^{e v}$ with $T_{s c^{-}}$ invariant differential. From the discussion above we have:

$$
H^{*}\left(\mathrm{CH}_{\text {loc }}^{\text {st }}\left(\mathcal{C}_{\|}\langle\vec{a}, 0\rangle\right)\right)=H^{0}\left(\mathrm{CH}_{\text {loc }}^{\text {st }}\left(\mathcal{C}_{\|}^{e v}\langle\vec{a}, 0\rangle\right)\right)=\chi\left(\mathrm{CH}_{\text {loc }}^{\text {st }}\left(\mathcal{C}_{\|}^{e v}\langle\vec{a}, 0\rangle\right)\right)=\chi\left(\mathbb{S}_{1}^{e v}\langle\vec{a}, 0\rangle\right),
$$

where $\mathbb{S}_{1}^{e v}$ is the version of $\mathbb{S}_{1}$ with t-twisted even component. There is well-defined image $\mathrm{K}\left(\mathcal{C}_{\|}\right)$of the complex inside of the $T_{s c}$-equivariant $K$-theory. Thus the Euler characteristics of the LHS of last formula can be computed within $K_{T_{s c}}\left(\mathrm{FHilb}^{\text {free }}\right)$ and here we can use the analog Negut's theorem for the push-forward along the fibers of the projection

Proposition 8.6.4. For any rational function $r\left(\mathcal{L}_{n}\right)$ with coefficients rational functions of $\mathcal{L}_{i}, i<n$, the $K$-theory push-forward is given by

$$
\pi_{*}\left(r\left(\mathcal{L}_{n}\right)\right)=\int \frac{r(z)}{\left(1-z^{-1}\right)} \prod_{i=1} \zeta^{\prime}\left(\mathcal{L}_{i} / z\right) \frac{d z}{z}
$$

where the contour of integration separates the set $\operatorname{Poles}(r(z)) \bigcup\{0, \infty\}$ from the poles of the rest of the integrant.

The $K$-theory class of the complex $\mathrm{CH}_{\text {loc }}^{\text {st }}\left(\mathcal{C}_{\|}^{e v}\right)$ is $\prod_{1 \leqslant i<j \leqslant n}\left(1-q t \mathcal{L}_{i} / \mathcal{L}_{j}\right)$. Hence we can apply the formula from the previous proposition iterative to obtain the iterated residue integral formula for the desired link invariant:

$$
\int \ldots \int \prod_{i} \frac{z_{i}^{b_{i}}\left(1+a z_{i}^{-1}\right)}{1-z^{-1}} \prod_{1 \leqslant i<j \leqslant n} \zeta\left(\frac{z_{i}}{z_{j}}\right) \frac{d z_{1}}{z_{1}} \ldots \frac{d z_{n}}{z_{n}} .
$$


The final step of the proof is a delicate analysis of the iterated residue that was done in the work of Negut [Neg15] in the context of $K$-theory of the flag Hilbert scheme.

\section{REFERENCES}

[AK15a] Sergey Arkhipov and Tina Kanstrup. Braid group actions on matrix factorizations, 2015, 1510.07588 .

[AK15b] Sergey Arkhipov and Tina Kanstrup. Equivariant Matrix Factorizations and Hamiltonian reduction. Bull. of Korean Math. Soc., (5):1803-1825, 2015, 1510.07472v1

[CG97] Neil Chris and Victor Ginzburg. Representation Theory and Complex Geometry. Birkhauser, 1997.

[Dyc11] Tobias Dyckerhoff. Compact generators in categories of matrix factorizations. Duke Math. J., (2):223-274, 2011.

[EH16] Ben Elias and Matt Hogancamp. On the computation of torus link homology, 2016, 1603.00407.

[EH17a] Ben Elias and Matthew Hogancamp. Categorical diagonalization, 2017, 1707.04349v1.

[EH17b] Ben Elias and Matthew Hogancamp. Categorical diagonalization of full twists, 2017, 1801.00191v1.

[Eis80] David Eisenbud. Homological algebra on a complete intersection, with an application to group representations. Trans. Amer. Math. Soc., (1):35-64, 1980.

[GH17] Eugene Gorsky and Matthew Hogancamp. Hilbert schemes and $y$-ification of Khovanov-Rozansky homology. 2017, 1712.03938v1

[GORS14] Eugene Gorsky, Alexei Oblomkov, Jacob Rasmussen, and Vivek Shende. Torus knots and the rational DAHA. Duke Mathematical Journal, 163:2709-2794, 2014.

[GRN16] Eugene Gorsky, Jacob Rasmussen, and Andrei Negut. Flag Hilbert schemes, colored projectors and Khovanov-Rozansky homology, 2016, 1608.07308.

[Hai02] Mark Haiman. Vanishing theorems and character formulas for the hilbert scheme of points in the plane. Invent. Math., 149(2):371-407, 2002.

[HL19] Daniel Halpern-Leistner. in preparation, 2019.

[Isi13] Umut Isik. Equivalence of the Derived Category of a Variety with a Singularity Category. IMRN, (12):2728-2808, 2013, 1011.1484v1.

[Jon87] V. F. R. Jones. Hecke algebra representations of braid groups and link polynomials. The Annals of Mathematics, 126(2):335, Sep 1987. doi 10.2307/1971403.

[Kho00] Mikhail Khovanov. A categorification of the Jones polynomial. Duke Mathematical Journal, 101(3):359-426, Feb 2000. doi 10.1215/s0012-7094-00-10131-7.

[KR08] Mikhail Khovanov and Lev Rozansky. Matrix factorizations and link homology II. Geometry and Topology, 12:1387-1425, 2008.

[KR10] Anton Kapustin and Lev Rozansky. Three-dimensional topological field theory and symplectic algebraic geometry II. Communications in Number Theory and Physics, 4(3):463-549, 2010. doi 10.4310/cntp.2010.v4.n3.a1

[KRS09] Anton Kapustin, Lev Rozansky, and Natalia Saulina. Three-dimensional topological field theory and symplectic algebraic geometry I. Nuclear Physics B, 816(3):295-355, Aug 2009. doi $10.1016 /$ j.nuclphysb.2009.01.027.

[Neg15] A. Negut. Moduli of flags of sheaves and their K-theory. Algebraic Geometry, 2(1):19-43, Mar 2015. doi $10.14231 /$ ag-2015-002.

[OR17] Alexei Oblomkov and Lev Rozansky. HOMFLYPT homology of Coxeter links, 2017, 1706.00124v1.

[OR18a] Alexei Oblomkov and Lev Rozansky. 3D TQFT and HOMFLYPT homology, 2018, 1812.06340v1.

[OR18b] Alexei Oblomkov and Lev Rozansky. A categorification of a cyclotomic Hecke algebra, 2018, $1801.06201 \mathrm{v} 1$

[OR18c] Alexei Oblomkov and Lev Rozansky. Affine braid group, JM elements and knot homology. Transformation Groups, Jan 2018. doi 10.1007/s00031-018-9478-5

[OR18d] Alexei Oblomkov and Lev Rozansky. Categorical Chern character and braid groups, 2018, $1811.03257 \mathrm{v} 1$.

[OR18e] Alexei Oblomkov and Lev Rozansky. Knot homology and sheaves on the Hilbert scheme of points on the plane. Selecta Mathematica, 24(3):2351-2454, Jan 2018. doi 10.1007/s00029-017-0385-8

[OR18f] Alexei Oblomkov and Lev Rozasky. Categorical Chern character and Hall algebras. in preparation, 2018. 
[Orl04] Dmitri Orlov. Triangulated categories of singularities and D-branes in Landau-Ginzburg models. Proc. Steklov Inst. Math., 246(3):227-248, 2004.

[Orl09] Dmitri Orlov. Derived categories of coherent sheaves and triangulated categories of singularities. Algebra, Arithmetic, and Geometry, pages 503-531, 2009. doi 10.1007/978-0-8176-4747-6_16

[Orl11] Dmitri Orlov. Matrix factorizations for nonaffine LG-models. Mathematische Annalen, 353(1):95108, May 2011. doi $10.1007 /$ s00208-011-0676-x

[ORS18] Alexei Oblomkov, Jacob Rasmussen, and Vivek Shende. The Hilbert scheme of a plane curve singularity and the HOMFLY homology of its link. Geometry and Topology, 22(2):645-691, Jan 2018. doi $10.2140 /$ gt.2018.22.645

[PV11] Alexander Polishchuk and Arkady Vaintrob. Matrix factorizations and singularity categories for stacks. Annales de linstitut Fourier, 61(7):2609-2642, 2011. doi 10.5802/aif.2788.

[Soe01] Wolfgang Soergel. Langlands' philosophy and Koszul duality. In Algebra-Representation Theory (Constanta,2000), pages 379-414, 2001.

A. Oblomkov, Department of Mathematics and Statistics, University of Massachusetts at Amherst, Lederle Graduate Research Tower, 710 N. Pleasant Street, Amherst, MA 01003 USA E-mail address: oblomkov@math.umass.edu 